\title{
DIRICHLET PROBLEM IN AN ANGULAR DOMAIN WITH RAPIDLY OSCILLATING BOUNDARY: MODELING OF THE PROBLEM AND ASYMPTOTICS OF THE SOLUTION
}

\author{
S. A. NAZAROV
}

\begin{abstract}
Leading asymptotic terms are constructed and justified for the solution of the Dirichlet problem corresponding to the Poisson equation in an angular domain with rapidly oscillating boundary. In addition to an exponential boundary layer near the entire boundary, a power-law boundary layer arises, which is localized in the vicinity of the corner point. Modeling of the problem in a singularly perturbed domain is studied; this amounts to finding a boundary-value problem in a simpler domain whose solution approximates that of the initial problem with advanced precision, namely, yields a two-term asymptotic expression. The way of modeling depends on the opening $\alpha$ of the angle at the corner point; the cases where $\alpha<\pi, \alpha \in(\pi, 2 \pi)$, and $\alpha=2 \pi$ are treated differently, and some of them require the techniques of selfadjoint extensions of differential operators.
\end{abstract}

\section{§1. Setting of the PRoblem}

On the plane $\mathbb{R}^{2}$, consider a domain $\Omega$ bounded by a simple closed contour $\Gamma$ that is smooth everywhere except a point $\mathcal{O}$. Near $\mathcal{O}$, the domain $\Omega$ coincides with an angle $\mathbb{K}$ of opening $\alpha \in(0,2 \pi]$. We fix Cartesian coordinates $x=\left(x_{1}, x_{2}\right)$ and polar coordinates $(r, \varphi)$ centered at $\mathcal{O}$; the $x_{1}$-axis and the polar axis are directed along the bisector of the angle $\mathbb{K}=\{x: r>0,|\varphi|<\alpha / 2\}$.

Let $l$ be the length of the contour $\Gamma$, let $N$ be a large positive integer, and let $\varepsilon=$ $(2 \pi N)^{-1} l$ be a small parameter. We introduce a smooth (for simplicity), periodic, ${ }^{1}$ and nonnegative function $H \neq 0$. Outside the disk $\mathbb{B}_{\varepsilon R}=\{x: r<\varepsilon R\}$ we define a rapidly oscillating $\operatorname{arc} \Gamma(\varepsilon)$ by the formula

$$
\Gamma(\varepsilon) \backslash \mathbb{B}_{\varepsilon R}=\left\{x \in \mathcal{V}_{d} \backslash \mathbb{B}_{\varepsilon R}: n=\varepsilon H\left(\varepsilon^{-1} s\right)\right\},
$$

where $\mathcal{V}_{d}=\{x \in \bar{\Omega} \backslash \mathcal{O}: \operatorname{dist}(x, \partial \Omega)<R d\}$ is a curvilinear strip with sharp extremities, which adjoins the arc $\Gamma \backslash \mathcal{O}$. The number $d>0$ is chosen so that the natural curvilinear cooridnates $(n, s)$ are defined on $\mathcal{V}_{d}$; here $s$ is the arclength on $\Gamma$, measured counterclockwise from the point $\mathcal{O}$, and $n$ is the oriented distance to $\Gamma, n>0$ inside $\Gamma$. In the disk $\mathbb{B}_{2 \varepsilon R}$, we use the dilated coordinates $\xi=\varepsilon^{-1} x$; it is assumed (again for simplicity) that the set $\left\{\xi \in \mathbb{R}^{2}: x=\varepsilon \xi \in \Gamma(\varepsilon) \cap \mathbb{B}_{2 \varepsilon R}\right\}$ does not depend on $\varepsilon$ and is a smooth arc included in the angle $\overline{\mathbb{K}} \backslash \mathcal{O}$.

2000 Mathematics Subject Classification. Primary 35B40, 35J25.

Key words and phrases. Dirichlet problem, oscillating boundary, corner point, asymptotics, selfadjoint extension.

Supported by RFBR (grant 06-01-257).

${ }^{1}$ All periodic functions in this paper have period $2 \pi$. 


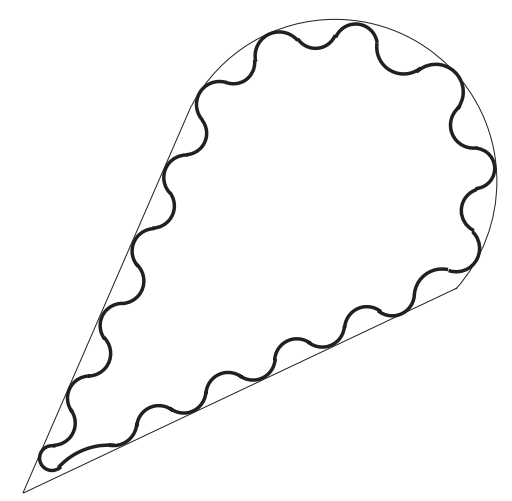

Figure 1

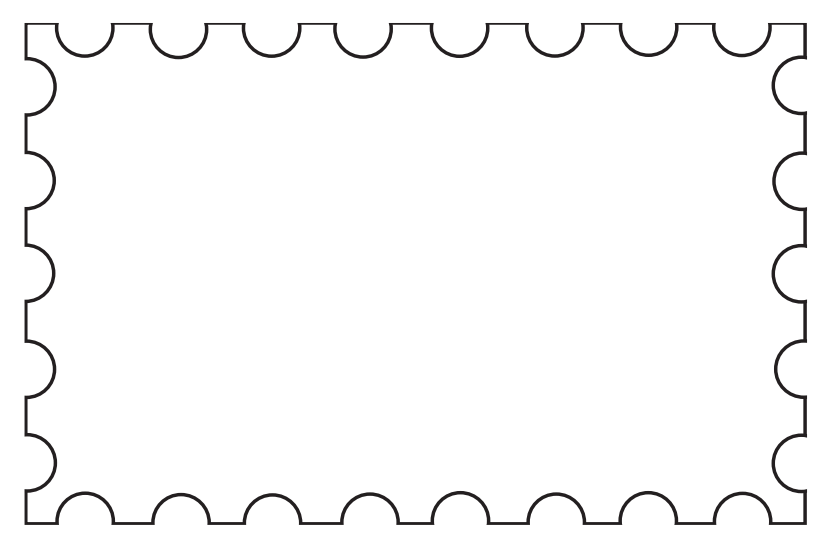

FIGURE 2

Let $\Omega(\varepsilon)$ denote the domain bounded by $\Gamma(\varepsilon)$ (see Figure 1). We consider the Dirichlet problem

$$
-\Delta_{x} u_{\varepsilon}(x)=f(x), x \in \Omega(\varepsilon), \quad u_{\varepsilon}(x)=0, x \in \Gamma(\varepsilon),
$$

where $f$ is a function defined on $\Omega$, and hence, on the domains $\Omega(\varepsilon) \subset \Omega$ with $\varepsilon \in\left(0, \varepsilon_{0}\right]$, $\varepsilon_{0}>0$. If $f \in L_{2}(\Omega)$, problem $(2)$ admits a solution $u_{\varepsilon} \in H^{2}(\Omega(\varepsilon)) .^{2}$

Our main goal in this paper consists in modeling the above problem, i.e., in finding a simpler domain and posing a boundary-value problem the solution of which approximates $u^{\varepsilon}$ with an acceptable accuracy. This is done with the help of asymptotic formulas. In the case of a smooth contour, the asymptotics of the solution of the problem in a domain with periodic boundary was constructed in the papers [1]-[8] and elsewhere. In addition to the regular expansion

$$
u_{0}(x)+\varepsilon u^{\prime}(x)+\varepsilon^{2} u^{\prime \prime}(x)+\cdots,
$$

\footnotetext{
${ }^{2}$ It would be correct to talk about families of boundary-value problems and their solutions, but for us, $\varepsilon$ is a small but fixed parameter, so we name the objects in the singular.
} 


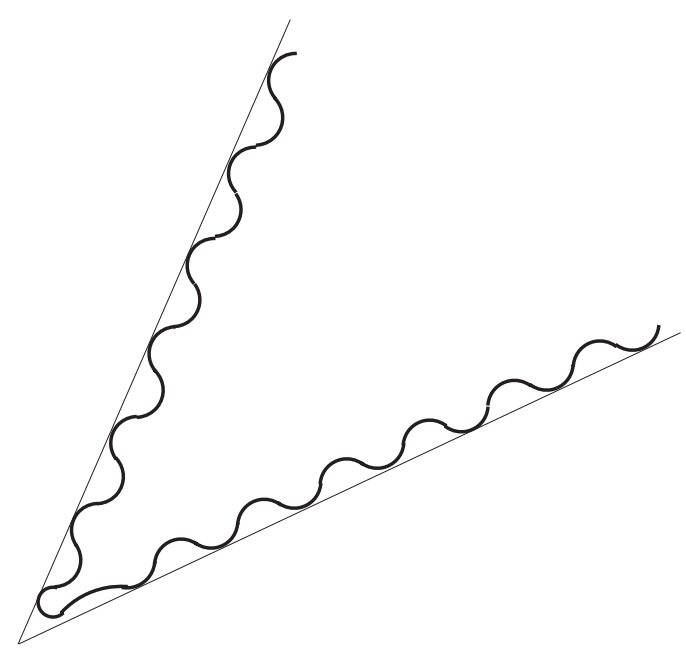

FiguRE 3

the terms of which are the solutions of Dirichlet problems in the fixed domain $\Omega$, we have, near the rapidly oscillating boundary $\left\{x: n=\varepsilon H\left(\varepsilon^{-1} s\right), s \in \Gamma\right\}$, an exponential boundary layer, which is described in terms of $\eta_{2}$-periodic solutions of the limiting problem in the half-strip $\Pi=\left\{\eta \in \mathbb{R}^{2}: \eta_{2} \in(0,2 \pi), \eta_{1}>H\left(\eta_{2}\right)\right\}$ with curvilinear base (see Section 2 ). The modeling of problem (2) reduces to either imposing the so-called "wall boundary conditions" on $\partial \Omega$ (see, e.g., [3]-[6]), or to forming a regularly perturbed domain

$$
\Omega^{\prime}(\varepsilon)=\left(\Omega \backslash \mathcal{V}_{d}\right) \cup\left\{x \in \mathcal{V}_{d}: n>m_{0} \varepsilon\right\}
$$

and imposing the homogeneous Dirichlet conditions on the "smooth image" $\Gamma^{\prime}(\varepsilon)=$ $\partial \Omega^{\prime}(\varepsilon)$ of the rapidly oscillating boundary $\partial \Omega(\varepsilon)$ (see, e.g., $[9,3,10]$ ). In both cases, the solution of the model problem yields the correct two-term asymptotics (see (3)) of the function $u_{\varepsilon}$ and serves as an advanced accuracy approximation in the $L_{2}$-metric to the solution of problem (2) (the order of the $H^{1}$-norm of the boundary layer is smaller than that of the correction $u^{\prime}$ of regular type, and therefore, is not small in the energy metric). We emphasize that for many applications it suffices to have even this, weak, asymptotic approximation.

If the contour $\Gamma$ is piecewise smooth (see Figures 1 and 2), the construction of the asymptotics for the solution of (2) becomes more complicated, involving a power-law boundary layer near corner points; the latter is described in terms of solutions of a new limiting problem in a domain $\Xi$ that goes to infinity as an angle with periodically perturbed sides (see Figure 3).

For such unbounded domains, the solvability of Dirichlet problems for formally selfadjoint systems of differential equations and the asymptotics of their solutions near infinity were studied in [11]; in Section 3 we present the results required for our purposes in the present paper, restricting ourselves to the Dirichlet problem for the Laplace operator.

The algorithm itself of constructing the asymptotic expansion with respect to the small parameter $\varepsilon$ is well known. Already at the beginning of the $1980 \mathrm{~s}$, two equivalent methods were developed, based on compound and on matched asymptotic expansions (see [12]-[15] and [16, 17], respectively, and also many other publications on this topic).

The asymptotic expansions at infinity for solutions in an unbounded domain are multiscaled: they include power-law solutions typical of angular domains, together with boundary layers related to the periodic structure of the contour $\partial \Xi$ (see formulas (15)-(17) 
below). For this reason, the procedure of rearrangement of discrepancies, used in the method of compound expansions, is applied not only to power-law solutions, but also to boundary layers. This version of the procedure was presented in detail in the paper [15], in the context of regularly degenerating elliptic problems with a small parameter at the highest order derivatives, either in the differential equations or in the boundary conditions. It should be emphasized that in the framework of the method of matched expansions, the boundary layer type terms match automatically, because the power-law solutions do so.

In Sections 4 and 5 of the present paper we construct and justify the leading terms of the asymptotics for the solution of problem (2). Two cases should be distinguished: the outgoing angle $(\alpha<\pi)$ and the incoming angle $(\alpha \in(\pi, 2 \pi])$. The reason is that in the latter case the solution $u_{0}$ of the limiting problem in $\Omega$ is not as smooth as needed, so that the power-law boundary layer near the point $\mathcal{O}$ becomes of principal importance. The asymptotic construction in Section 4 is simple (two terms of regular type plus an exponential boundary layer smoothed near the corner point), and its justification leans upon the basic (energy) inequality (see [18]). The asymptotics in Section 5 is based on the method of matched expansions, but this time justification involves the constructions (55) or (56) that come from the method of compound expansions. Moreover, we are forced to estimate the asymptotic remainder in the scale of weighted spaces, because in the case of an incoming angle the energy estimate turns out to be inaccurate, and hence useless for modeling of problem (2).

The last three sections of the paper are devoted to modeling itself. In Section 6 we show that the incoming angle does not bring any changes in the model, as compared to the case of a smooth contour $\Gamma$ (see Remark 5 and Proposition 1). However, because of the imposed requirement $\Omega(\varepsilon) \subset \Omega$, the wall boundary conditions (see [3]-[6]) do not ensure the unique solvability of the model problem, and we prefer to use the "smooth image" principle for the rapidly oscillating boundary; in our case this image has a corner point. In Section 7, when treating the case of an incoming angle, we encounter a new difficulty: both boundary layers remain small in the $L_{2}(\Omega(\varepsilon)$ )-norm, but the regular type term involves a weight function $\zeta \in L_{2}(\Omega)$, which is the solution of the homogeneous Dirichlet problem in $\Omega$, and this term has a singularity at the vertex $\mathcal{O}$ and fails to belong to the energy class $H^{1}(\Omega)$ (see formulas (10) and (9) below). For this reason, modeling of problem (2) invokes the machinery of selfadjoint extensions of differential operators.

Selfadjoint extensions have been used in this context for a description of the far field (the outer expansion in the framework of the matched expansions method) in "dirty" media, i.e., in homogeneous media that may have small defects or inclusions; for the first time this was done, apparently, in [19]. The approach has then been elaborated by various mathematicians in St. Petersburg (see the survey [20]). In the paper [21], a relationship was found between the method of matched expansions and the theory of selfadjoint extensions; in particular, this relationship provides proper settings of nonstandard boundary-value problems that arise in asymptotic analysis. On this way, in [21] it was shown how to find the parameters of the appropriate selfadjoint extensions. Also in [21], asymptotically sharp estimates were obtained for the discrepancies caused by modeling the defects with the help of "potentials with zero radius." In Section 7, similar results are obtained for problem (2) in an angular domain with rapidly oscillating boundary.

In Section 8 we consider the special case where $\alpha=2 \pi$, i.e., where $\Omega$ has a cut (cf. Figure 4). By using the concept of a "plane image" of a crack, suggested in [22, 23] for cracks through thin 3-dimensional plates, the model can be constructed without invoking selfadjoint extensions: it suffices to choose the length of the cut appropriately. 


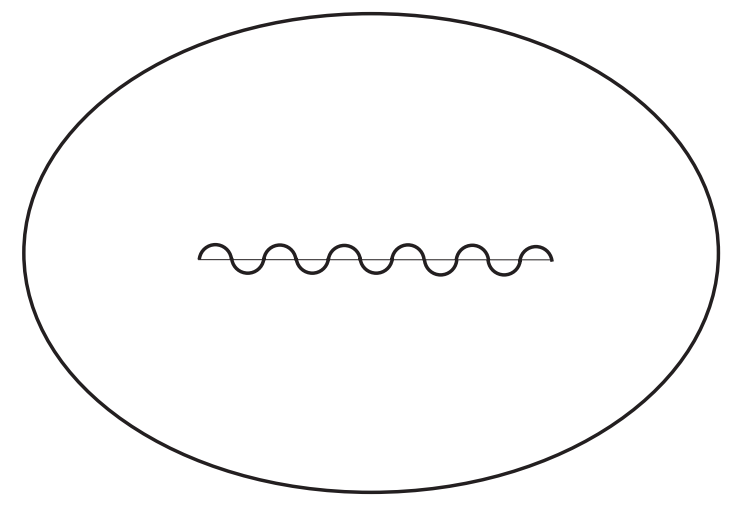

FIGURE 4
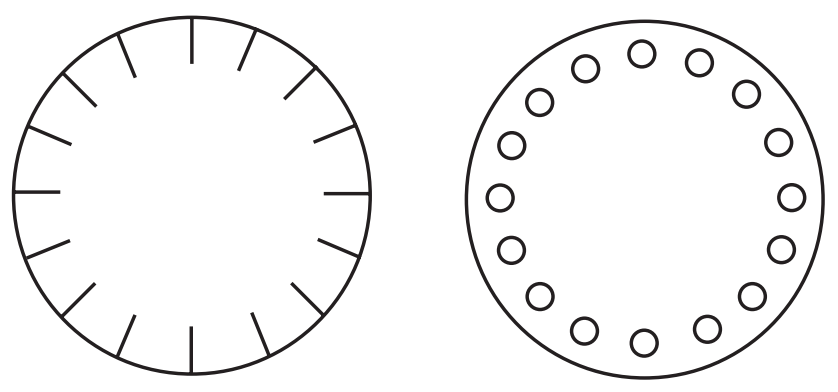

FiguRE 5

For clarity, we consider the simplest setting, dealing with the Poisson equation and a single corner point on the contour $\Gamma$. Of course, many generalizations are straightforward, especially the passage to several corner points (see Figures 2 and 4): this only requires bulkier notation in the construction of asymptotics and in the listing of all possible selfadjoint extensions. The rapidly oscillating boundary under consideration need not be the graph of a smooth periodic function - all calculations below remain valid if the function $H$ in (1) depends smoothly on the slow variable $s$, or is piecewise smooth relative to the fast variable $\varepsilon^{-1} s$. Other types of locally periodic perturbations of the boundary can also be treated (Figure 5).

\section{$\S 2$. The EXPONENTIAL BOUNDARY LAYER}

Should the boundary $\partial \Omega$ be smooth, we would be able to restrict the construction of the asymptotics for $u_{\varepsilon}$ to the study of two limiting problems, namely, the Dirichlet problem for the Poisson equation in $\Omega$ and the following problem in the half-strip $\Pi=$ $\left\{\eta \in \mathbb{R}^{2}: \eta_{1}>H\left(\eta_{2}\right), \eta_{2} \in(0,2 \pi)\right\}$ with curvilinear base $\gamma=\left\{\eta \in \partial \Pi: \eta_{2} \in(0,2 \pi)\right\}:$

$$
\begin{gathered}
-\Delta_{\eta} w(\eta)=F(\eta), \quad \eta \in \Pi, \quad w(\eta)=G(\eta), \quad \eta \in \gamma, \\
w\left(\eta_{1}, 0\right)=w\left(\eta_{1}, 2 \pi\right), \quad \frac{\partial w}{\partial \eta_{2}}\left(\eta_{1}, 0\right)=\frac{\partial w}{\partial \eta_{2}}\left(\eta_{1}, 2 \pi\right), \quad \eta_{1}>H(0) .
\end{gathered}
$$

Problem (4) arises when we dilate the coordinates $x \mapsto \eta=\left(\varepsilon^{-1} n, \varepsilon^{-1} s\right)$ and formally pass to $\varepsilon=0$; the periodicity conditions on the lateral sides of the half-strip come from the geometric structure of the contour $\Gamma(\varepsilon)$. 
The next statement (Lemma 1) is ensured, e.g., by the theory of elliptic boundaryvalue problems in domains with piecewise smooth boundary (see the key publications [24]-[26] and, e.g., the book [27]). We denote by $H_{\text {per }}^{l}(\Pi)$ the Sobolev space of functions periodic in the variable $\eta_{2}$, and by $H_{\mathrm{per}}^{l+1 / 2}(\gamma)$ the corresponding Sobolev-Slobodetskiu space.

Lemma 1. Suppose $l \in \mathbb{N}=\{1,2, \ldots\}, e^{\beta \eta_{1}} F \in H_{\text {per }}^{l-1}(\Pi), G \in H_{\text {per }}^{l+1 / 2}(\gamma)$, and $\beta \in$ $(0,1)$. Problem (4) admits a solution $w \in H_{\mathrm{per}}^{l+1}(\Pi)$ that decays at infinity if and only if

$$
\int_{\Pi} F(\eta) z(\eta) d \eta+\int_{\gamma} G(\eta) \partial_{\nu(\eta)} z(\eta) d s_{\eta}=0
$$

Here $\partial_{\nu(\eta)}$ is the derivative along the inward normal to the arc $\gamma \subset \partial \Pi$, $d s_{\eta}$ is the arclength element, and $z(\eta)=\eta_{1}-z_{0}(\eta)$ is a solution of (4) growing at infinity, $z_{0}$ being a bounded solution of problem (4) with $F(\eta)=0$ and $G(\eta)=\eta_{1}$. The solution $w \in H_{\mathrm{per}}^{l+1}(\Pi)$ is unique. Under the solvability conditions (5) we have $e^{\beta \eta_{1}} w \in H_{\mathrm{per}}^{l+1}(\Pi)$ and

$$
\left\|e^{\beta \eta_{1}} w ; H_{\mathrm{per}}^{l+1}(\Pi)\right\| \leq c\left(\left\|e^{\beta \eta_{1}} F ; H_{\mathrm{per}}^{l+1}(\Pi)\right\|+\left\|G ; H_{\mathrm{per}}^{l+1 / 2}(\gamma)\right\|\right) .
$$

The bounded, harmonic, and periodic function $z_{0}$ mentioned in Lemma 1 satisfies

$$
z_{0}(\eta)=m(\Pi)+\widetilde{z}(\eta), \quad\left|\nabla_{\eta}^{k} \widetilde{z}(\eta)\right| \leq c_{k} e^{-\eta_{1} / 2}, \quad k \in \mathbb{N}_{0}=\{0,1,2, \ldots\} .
$$

Lemma 2. The constant $m(\Pi)$ in (6) is positive.

Proof. Applying the method of [25], we use the Green formula to obtain the identities

$$
\begin{aligned}
\int_{\gamma} z_{0} \partial_{\nu(\eta)} z d s_{\eta} & =\left.\lim _{T \rightarrow \infty} \int_{0}^{2 \pi}\left(z_{0}\left(1-\frac{\partial z_{0}}{\partial \eta_{1}}\right)-\left(\eta_{1}-z_{0}\right) \frac{\partial z_{0}}{\partial \eta_{1}}\right)\right|_{\eta_{1}=T} d s_{\eta}=m(\Pi), \\
\int_{\gamma} z_{0} \partial_{\nu(\eta)} z d s_{\eta} & =\int_{\gamma} \eta_{1} \partial_{\nu(\eta)} \eta_{1} d s_{\eta}-\int_{\gamma} z_{0} \partial_{\nu(\eta)} z_{0} d s_{\eta} \\
& =\int_{0}^{2 \pi} H\left(\eta_{2}\right) d \eta_{2}+\int_{\Pi}\left|\nabla_{\eta} z_{0}\right|^{2} d \eta
\end{aligned}
$$

In the first of them, we have used formula (6) to calculate the limit. The right-hand side in the second identity is positive for obvious reasons.

\section{§3. THE POWER-LAW BOUNDARY LAYER}

If $\alpha<\pi$ and $f \in L_{2}(\Omega)$, then the weak solution $u_{0} \in \stackrel{\circ}{H}^{1}(\Omega)$ of the limiting $(\varepsilon=0)$ problem

$$
-\Delta_{x} u_{0}(x)=f(x), \quad x \in \Omega, \quad u_{0}(x)=0, x \in \partial \Omega,
$$

belongs to the Sobolev space $H^{2}(\Omega)$. Moreover, the products $r^{-2} u_{0}$ and $r^{-1} \nabla_{x} u_{0}$ lie in the Lebesgue space $L_{2}(\Omega)$ (see, e.g., the introductory Chapter 2 in the book [27]). If $\alpha>\pi$, then the solution $u_{0}$ becomes nonclassical and acquires the following singularity at the point $\mathcal{O}$ :

$$
u_{0}(x)=\chi(r) K r^{\pi / \alpha} \cos (\pi \varphi / \alpha)+\widetilde{u}_{0}(x) .
$$

Here $\chi \in C_{0}^{\infty}(\mathbb{R})$ is a cut-off function with $\chi(r)=1$ for $r<R^{0} / 2$ and $\chi(r)=0$ for $r>R^{0}$, and the radius $R^{0}$ is chosen so that $\partial \Omega \cap \mathbb{B}_{R^{0}}=\partial \mathbb{K} \cap \mathbb{B}_{R^{0}}$. The remainder term $\widetilde{u}_{0}$ falls into the space $H^{2}(\Omega)$, and the intensity coefficient $K$ can be found by the formula (see [25])

$$
K=\int_{\Omega} f(x) \zeta(x) d x
$$


where $\zeta \in L_{2}(\Omega)$ is a harmonic function in $\Omega$ that vanish on $\partial \Omega$ and admits the representation

$$
\zeta(x)=\pi^{-1} \chi(r) r^{-\pi / \alpha} \cos (\pi \varphi / \alpha)+\zeta_{0}(x), \quad \zeta_{0} \in \stackrel{\circ}{H^{1}}(\Omega) .
$$

As will be shown in Section 4 , in the case where $\alpha<\pi$ it is possible to construct and justify the two leading terms of the asymptotics for the solution $u_{\varepsilon}$ of problem (2) with the help of solutions of the limiting problems (7) and (4). However, for $\alpha>\pi$, to remove the singularities of the second derivatives of the function (8), which are not square integrable, we need to construct an additional boundary layer near the corner point $\mathcal{O}$. Namely, in this case the asymptotic constructions invoke solutions of yet another limiting problem

$$
-\Delta_{\xi} v(\xi)=\mathcal{F}(\xi), \quad \xi \in \Xi, \quad v(\xi)=\mathcal{G}(\xi), \quad \xi \in \partial \Xi,
$$

where $\Xi$ is the domain obtained from the singularly perturbed domain $\Omega(\varepsilon)$ by the coordinate dilation $x \mapsto \xi=\varepsilon^{-1} x$ followed by the passage to $\varepsilon=0$ (see Section 1 and Figure 3 ). Off the disk $\mathbb{B}_{2 R}$, the boundary $\partial \Xi$ is formed by two half-infinite periodic curves described by the equations $\xi_{2}^{ \pm}=H\left(\xi_{1}^{ \pm}\right)$; here the $\xi^{ \pm}$are the Cartesian coordinates with axes $\xi_{2}^{ \pm}$ and $\xi_{1}^{ \pm}$directed, respectively, along the side $\Gamma^{ \pm}=\left\{\xi \in \mathbb{R}^{2}: \rho=|\xi|>0, \varphi= \pm \alpha / 2\right\}$ of the angle $\mathbb{K}$ and inward that angle. In other words, the domain $\Xi$ has an angular exit to infinity, with a periodic boundary. For domains of such a form, the Dirichlet problem for elliptic selfadjoint system of second order differential equations was studied in [11]. We formulate two results of [11] that concern solvability and asymptotics, as applied to the scalar problem (11). For this, we shall need the function space $\mathbf{V}_{\beta, \gamma}^{l}(\Xi)$ with double weight, introduced in [11], that consists of all functions $y \in H_{\mathrm{loc}}^{l}(\bar{\Xi})$ for which the norm

$$
\left\|y ; \mathbf{V}_{\beta, \gamma}^{l}(\Xi)\right\|=\left(\sum_{k=0}^{l}\left\|(1+\rho)^{\beta-l+k} \Theta^{\gamma-l+k} \nabla_{\xi}^{k} y ; L_{2}(\Xi)\right\|^{2}\right)^{1 / 2}
$$

is finite, where $(\rho, \varphi)$ are polar coordinates, $\Theta(\xi)=\left(\left(1+\rho^{2}\right)^{-1}+(|\alpha|-\varphi)^{2}\right)^{1 / 2}$, and $\nabla_{x}^{k} y$ is the collection of all partial derivatives of $y$ of order $k$. Since in the $d$-neighborhood of the sides of $\mathbb{K}$ we have $\Theta(\xi)=O\left((1+\rho)^{-1}\right)$, and the total weight $O\left((1+\rho)^{\beta-\gamma}\right)$ in the norm (12) does not depend on the order $k$ of the derivatives of $y$, the trace space $\mathbf{V}_{\beta, \gamma}^{l-1 / 2}(\partial \Xi)$ with $l \in \mathbb{N}$ can be endowed with the Sobolev-Slobodetskil weight norm

$$
\left\|y ; \mathbf{V}_{\beta, \gamma}^{l-1 / 2}(\partial \Xi)\right\|=\left\|\left(1+\rho^{2}\right)^{(\beta-\gamma) / 2} y ; H^{l-1 / 2}(\partial \Xi)\right\| .
$$

Theorem 1. 1) Suppose $l \in \mathbb{N},|\beta-l|<\pi / \alpha,|\gamma-l|<1 / 2$, and

$$
\mathcal{F} \in \mathbf{V}_{\beta, \gamma}^{l-1}(\Xi), \quad \mathcal{G} \in \mathbf{V}_{\beta, \gamma}^{l+1 / 2}(\partial \Xi)
$$

Then problem (11) admits a unique solution $v \in \mathbf{V}_{\beta, \gamma}^{l+1}(\Xi)$ and

$$
\left\|v ; \mathbf{V}_{\beta, \gamma}^{l+1}(\Xi)\right\| \leq c\left(\left\|\mathcal{F} ; \mathbf{V}_{\beta, \gamma}^{l-1}(\Xi)\right\|+\left\|\mathcal{G} ; \mathbf{V}_{\beta, \gamma}^{l+1 / 2}(\partial \Xi)\right\|\right) .
$$

2) If the membership relations (13) are fulfilled with a larger weight index $\beta^{1} \in$ $(l+\pi / \alpha, l+\pi / \alpha+1 / 2)$, then the solution $v \in \mathbf{V}_{\beta, \gamma}^{l+1}(\Xi)$ of (11) can be written as ${ }^{3}$

$$
v(\xi)=C \rho^{-\pi / \alpha} \cos (\pi \varphi / \alpha)+\widetilde{v}(\xi),
$$

and the intensity factor $C$ and the remainder term $\widetilde{v} \in \mathbf{V}_{\beta^{1}, \gamma}^{l+1}(\Xi)$ satisfy the inequality

$$
|C|+\left\|\widetilde{v} ; \mathbf{V}_{\beta^{1}, \gamma}^{l+1}(\Xi)\right\| \leq c\left(\left\|\mathcal{F} ; \mathbf{V}_{\beta^{1}, \gamma}^{l-1}(\Xi)\right\|+\left\|\mathcal{G} ; \mathbf{V}_{\beta^{1}, \gamma}^{l+1 / 2}(\partial \Xi)\right\|\right)
$$

\footnotetext{
${ }^{3}$ In (14) and in (16) below we use the assumption $\Xi \subset \mathbb{K}$. It this fails, then the distinguished asymptotic terms should be multiplied by a suitable cut-off function.
} 
In what follows we shall need a special solution $Y$ of the homogeneous problem (11) with growth at infinity:

$$
Y(\xi)=\rho^{\pi / \alpha} \cos (\pi \varphi / \alpha)-Y_{0}(\xi) .
$$

Here $Y_{0}$ is the solution of problem (11) with right-hand sides $\mathcal{F}_{0}=0$ and $\mathcal{G}_{0}(\xi)=$ $\rho^{\pi / \alpha} \cos (\pi \varphi / \alpha)$. The latter cosine vanishes on the sides of $\mathbb{K}$. Therefore, $\mathcal{G}_{0}(\xi)=$ $O\left((1+\rho)^{-1+\pi / \alpha}\right)$ for $\xi \in \partial \Xi$, and we have $\mathcal{G}_{0} \in \mathbf{V}_{\beta, \gamma}^{l+1 / 2}(\partial \Xi)$ provided $2(\beta-\gamma-1+$ $\pi / \alpha)+1<0$. We can choose the weight indices $\beta$ and $\gamma$ so as to satisfy the above restriction and, simultaneously, the requirements of Theorem 1, 1), thus finding a solution $Y_{0} \in \mathbf{V}_{\beta, \gamma}^{l+1}(\Xi)$ of the problem mentioned above. Since the function $\xi \mapsto \rho^{\pi / \alpha} \cos (\pi \varphi / \alpha)$ does not belong to $\mathbf{V}_{\beta, \gamma}^{l+1}(\Xi)$, the solution (15) of the homogeneous problem (11) turns out to be nontrivial.

In the paper [11], the procedure of constructing the full asymptotic expansion as $|\xi| \rightarrow+0$ was described for the solutions of problem (11). Specifying this procedure, as applied to the solution $Y$, we write some asymptotic terms needed for what follows:

$$
\begin{aligned}
Y_{0}(\xi)= & -\frac{\pi}{\alpha}\left(m(\Pi) Y_{1}(\xi)-\sum_{ \pm} \chi_{ \pm}(\varphi) \rho^{-1+\pi / \alpha} \widetilde{z}\left(\xi^{ \pm}\right)\right) \\
& +m(\Xi) \rho^{-\pi / \alpha} \cos \left(\frac{\pi}{\alpha} \varphi\right)+\widetilde{Y}_{0}(\xi) .
\end{aligned}
$$

Here

$$
Y_{1}(\xi)= \begin{cases}\rho^{-1+\pi / \alpha}\left[\cos \left(\frac{1}{2}(\alpha-\pi)\right)\right]^{-1}\left[\cos \left(1-\frac{\pi}{\alpha}\right) \varphi\right], & \alpha \in(\pi, 2 \pi), \\ \frac{1}{\pi} \rho^{-1 / 2}\left[\log \rho \cos \left(\frac{\varphi}{2}\right)-\varphi \sin \left(\frac{\varphi}{2}\right)\right], & \alpha=2 \pi\end{cases}
$$

$\chi_{ \pm} \in C^{\infty}(\mathbb{R})$ is a cut-off function equal to 1 for $\pm \varphi \in(3 \alpha / 8, \alpha / 2)$ and to 0 for $\pm \varphi \in$ $(\alpha / 8, \alpha / 4), \widetilde{z}$ is the exponentially decaying part of the special solution of problem (4) in the half-strip $\Pi$ (see (6)), $m(\Pi)$ and $m(\Xi)$ are the respective characteristics of the domains $\Pi$ and $\Xi$ (see Lemma 2 and Remark 1), and the remainder term $\widetilde{Y}$ belongs to the weighted class $\mathbf{V}_{\widetilde{\beta}, \gamma}^{l+1}(\Xi)$ for any indices $l \in \mathbb{N}, \gamma \in(l-1 / 2, l+1 / 2)$, and $\widetilde{\beta}<l+\max \{2-\pi / \alpha, 2 \pi / \alpha\}$.

Remark 1 . The divergence of the integral

$$
\int_{\partial \Xi} \rho^{\pi / \alpha} \cos \left(\frac{\pi}{\alpha} \varphi\right) \frac{\partial}{\partial \nu(\xi)}\left(\rho^{\pi / \alpha} \cos \left(\frac{\pi}{\alpha} \varphi\right)\right) d s_{\xi}
$$

(the integrand is $\left.O\left(\rho^{-2(1-\pi / \alpha)}\right)\right)$ makes it impossible to repeat the calculations in Lemma 2 that have led to the inequality $m(\Pi)>0$. The results of [11] show that, to obtain an integral representation for the coefficient $m(\Pi)$, we must split off the expression $-\pi \alpha^{-1}(\ldots)$ on the right in formula (16), but then the possibility of checking the inequality $m(\Pi)>0$ would be lost.

Remark 2. Since the solution $Y$ of the homogeneous problem (11) is infinitely differentiable on the set $\bar{\Xi}$, we can construct its full asymptotic expansion at infinity and, in particular, estimate the maximum of the modulus of the remainder $\widetilde{Y}$ and of its gradient. The corresponding details can be found in [11]; here we present the result (to be used later) concerning the remainder $\widetilde{Y}$, together with a corollary of this result. Namely, the inequalities

$$
\begin{aligned}
\left|\widetilde{Y}_{0}(\xi)\right| & \leq c_{0} \rho^{-\max \{2-\pi / \alpha, 2 \pi / \alpha\}}, \\
\left|\nabla_{\xi} \widetilde{Y}_{0}(\xi)\right| & \leq c_{1}\left(\rho^{-1-\pi / \alpha}+\rho^{-2+\pi / \alpha}\left(\rho^{-1}+e^{\delta \rho(|\varphi|-\alpha / 2)}\right)\right)
\end{aligned}
$$


are true with positive constants $c_{i}$ and $\delta$. It should be noted that, for $\alpha=2 \pi$, the decay rate of $\widetilde{Y}$ is determined by the asymptotic term $C \rho^{-2 \pi / \alpha} \sin (2 \pi \varphi / \alpha)=C \rho^{-1} \sin \varphi$, which decays more slowly than the term $Y_{2}(\xi)=\rho^{-2+\pi / \alpha} \Phi(\varphi, \log \rho)=\rho^{-3 / 2} \Phi(\varphi, \log \rho)$ coming from the term $Y_{1}(\xi)$ and inheriting the dependence on $\log \rho$ from the latter. Therefore, the logarithm is absent in estimates (18) and (19). Moreover, we have taken into account the fact that the boundary layer that compensates for discrepancies left by the terms $C \rho^{-2 \pi / \alpha} \sin (2 \pi \varphi / \alpha)$ on the periodic part of the boundary, is of the order of $\rho^{-1-2 \pi / \alpha}$, so that there is no exponential factor in the first term on the right in (19). By (18), for $\alpha \in(\pi, 2 \pi)$ we have $\widetilde{Y} \in L_{2}(\Xi)$, and for $\alpha=2 \pi$ we have the relation

$$
\int_{\Xi \cap \mathbb{B}_{R}}|\widetilde{Y}(\xi)|^{2} d \xi=O(|\log R|), \quad R \rightarrow \infty .
$$

\section{$\S 4$. The LEADING ASYMPtotic TeRMS FOR $\alpha<\pi$}

The norm in the Kondrat'ev space (see [24]) is

$$
\left\|y ; V_{\beta}^{l}(\Omega)\right\|=\left(\sum_{k=0}^{l}\left\|r^{\beta-l+k} \nabla_{x}^{k} y ; L_{2}(\Omega)\right\|^{2}\right)^{1 / 2},
$$

where $l \in \mathbb{N}_{0}$ and $\beta \in \mathbb{R}$ are the smoothness and weight exponents. The space itself consists of all functions $y \in H_{\text {loc }}^{l}(\bar{\Omega} \backslash \mathcal{O})$ with finite norm (20). Suppose that

$$
f \in V_{\sigma}^{1}(\Omega), \quad \sigma \in(2-\pi / \alpha, 1]
$$

Since $V_{\sigma}^{1}(\Omega) \subset L_{2}(\Omega)$, there is a weak solution $u_{0} \in \stackrel{\circ}{H}^{1}(\Omega)$ of problem (7) lying in the class $V_{\sigma}^{3}(\Omega)$ (see, e.g., the introductory Chapter 2 in [27]), and

$$
\left\|u_{0} ; V_{\sigma}^{3}(\Omega)\right\| \leq c_{\sigma}\left\|f ; V_{\sigma}^{1}(\Omega)\right\|
$$

with a factor $c_{\sigma}$ growing infinitely as $\sigma \rightarrow 2-\pi / \alpha$.

Since $r^{\sigma} \nabla_{x}^{3} u_{0}, r^{\sigma-1} \nabla_{x}^{2} u_{0} \in L_{2}(\Omega)$, for the trace $\partial_{n} u_{0}(0, s)$ of the normal derivative on the arc $\Gamma$ we have

$$
\left\|r^{\sigma-1 / 2} \partial_{s} \partial_{n} u_{0} ; L_{2}(\Gamma)\right\|+\left\|r^{\sigma-3 / 2} \partial_{n} u_{0} ; L_{2}(\Gamma)\right\| \leq c\left\|f ; V_{\sigma}^{1}(\Omega)\right\| .
$$

For $\varkappa \in[0,1]$, let $\chi_{\varepsilon}^{\varkappa}$ denote the cut-off function equal to 1 in the neighborhood $\mathcal{V}_{\varepsilon^{\varkappa} d / 2}$ of the arc $\Gamma \backslash \mathcal{O}$ and to 0 off the set $\mathcal{V}_{\varepsilon^{\varkappa}}$; of course, this function can be chosen so as to satisfy

$$
0 \leq \chi_{\varepsilon}^{\varkappa}(x) \leq 1, \quad\left|\nabla_{x} \chi_{\varepsilon}^{\varkappa}(x)\right| \leq c \varepsilon^{-1} r^{-1} .
$$

If $\varkappa=0$, then there is no dependence on $\varepsilon$, and the cut-off function $\chi_{\varepsilon}^{0}$ is denoted by $\chi^{0}$. The size $d$ is selected so that $\chi_{\varepsilon}^{1}=1$ on the set $\partial \Omega(\varepsilon) \backslash \mathbb{B}_{\varepsilon R}$.

Estimate (23) and the restriction (21) on the weight index $\sigma$ imply that the product $\chi^{0} \partial_{n} u_{0}(0, \cdot)$ belongs to the energy class $H^{1}(\Omega)$. Therefore, the problem

$$
-\Delta_{x} u^{\prime}(x)=0, x \in \Omega(\varepsilon), \quad u^{\prime}(x)=\partial_{n} u_{0}(0, s), x \in \Gamma(\varepsilon),
$$

has a unique weak solution $u^{\prime} \in H^{1}(\Omega)$ (see the textbook [18]). The right-hand side of the boundary condition in $(25)$ is the trace on $\partial \Omega$ of a function of class $V_{\sigma}^{2}(\Omega)$, and since $\alpha \in(0, \pi)$ and $\sigma-1 \in(-\pi / \alpha, \pi / \alpha)$, the results of [27, Chapter 2] imply that $u^{\prime}$ falls into the class $V_{\sigma}^{2}(\Omega)$ and satisfies the estimate

$$
\left\|u^{\prime} ; V_{\sigma}^{2}(\Omega)\right\| \leq c\left\|u_{0} ; V_{\sigma}^{3}(\Omega)\right\| \leq c\left\|f ; V_{\sigma}^{1}(\Omega)\right\| .
$$


Using the Sobolev embedding theorem, $H^{2} \subset C$, and the corollaries of the 1-dimensional Hardy inequality,

$$
\begin{aligned}
\int_{0}^{\delta} t^{-2 \varkappa-1}|V(t)-V(0)|^{2} d t & \leq \frac{1}{\varkappa^{2}} \int_{0}^{\delta} t^{-2 \varkappa+1}\left|\frac{\partial V}{\partial t}(t)\right|^{2} d t \\
\frac{1}{\delta} \int_{0}^{\delta}|V(t)|^{2} d t & \leq C \int_{0}^{\delta}\left(\left|\frac{\partial V}{\partial t}(t)\right|^{2}+|V(t)|^{2}\right) d t
\end{aligned}
$$

where $V \in C^{1}[0,1], \delta \in(0, d)$, and $\varkappa>0$, we obtain the relations $(28)$

$$
\begin{gathered}
u_{0}(x)=\chi^{0}(x) n \partial_{n} u_{0}(0, s)+\widehat{u}_{0}(x), \quad u^{\prime}(x)=\chi^{0}(x) \partial_{n} u_{0}(0, s)+\widehat{u}^{\prime}(x), \\
\frac{1}{\delta} \int_{\Omega \cap \mathcal{V}_{\delta}} r^{2 \sigma-6}\left[\varrho(x)^{-4}\left|\widehat{u}_{0}(x)\right|^{2}+r^{2} \varrho(x)^{-2}\left(\left|\widehat{u}^{\prime}(x)\right|^{2}+\left|\nabla_{x} \widehat{u}_{0}(x)\right|^{2}\right)+r^{4}\left|\nabla_{x} \widehat{u}^{\prime}(x)\right|^{2}\right] d x \\
\leq c\left(\left\|u_{0} ; V_{\sigma}^{3}(\Omega)\right\|^{2}+\left\|u^{\prime} ; V_{\sigma}^{2}(\Omega)\right\|^{2}\right) \leq c\left\|f ; V_{\sigma}^{1}(\Omega)\right\|^{2} .
\end{gathered}
$$

Here $\varrho$ is a function subject to the estimate

$$
\begin{array}{ll}
c \varrho(x) \leq \operatorname{dist}(x, \partial \Omega) \leq C \varrho(x), & x \in \Omega \backslash \mathbb{B}_{R^{0}}, \\
c \varrho(x) \leq|x|^{-1} \operatorname{dist}(x, \partial \Omega) \leq C \varrho(x), & x \in \Omega \cap \mathbb{B}_{R^{0}},
\end{array}
$$

with positive constants $c$ and $C$.

An asymptotic approximation to the solution of problem (2) will be taken in the form

$$
U_{\varepsilon}(x)=u_{0}(x)-\varepsilon m(\Pi) u^{\prime}(x)-\varepsilon\left(1-\chi\left(\varepsilon^{-1} r\right)\right) \chi^{0}(x) \partial_{n} u_{0}(0, s) \widetilde{z}\left(\varepsilon^{-1} n, \varepsilon^{-1} s\right),
$$

where $u_{0}$ and $u^{\prime}$ are the solutions of (7) and (25), and $\widetilde{z}$ is a term of the boundary layer type, decaying exponentially at infinity in accordance with (6). Also, we introduce a bulkier intermediate asymptotic approximation:

$$
\begin{aligned}
\mathcal{U}_{\varepsilon}(x)=\left(1-\chi\left(\varepsilon^{-1} r\right)\right)\left[( 1 - \chi _ { \varepsilon } ^ { 1 } ( x ) ) \left(\widehat{u}_{0}(x)\right.\right. & \left.-\varepsilon m(\Pi) \widehat{u}^{\prime}(x)\right) \\
& \left.+\varepsilon \chi^{0}(x) \partial_{n} u_{0}(0, s) z\left(\varepsilon^{-1} n, \varepsilon^{-1} s\right)\right] .
\end{aligned}
$$

Due to the presence of the cut-off functions $1-\chi\left(\varepsilon^{-1} r\right)$ and $1-\chi_{\varepsilon}^{1}(x)$, and by the definition of the periodic harmonic function $z$ (see Lemma 1), expression (31) vanishes on the boundary $\partial \Omega(\varepsilon)$.

Lemma 3. We have

$$
\left\|\mathcal{U}_{\varepsilon}-U_{\varepsilon} ; H^{1}(\Omega(\varepsilon))\right\| \leq c \varepsilon^{\min \{2-\sigma, 3 / 2\}}\left\|f ; V_{\sigma}^{1}(\Omega)\right\|,
$$

where $c$ is a constant depending neither on $\varepsilon \in\left(0, \varepsilon_{0}\right]$ nor on the right-hand side $f$ of problem (7).

Proof. By (6) and the definition of $z$, we have

$$
\varepsilon z\left(\varepsilon^{-1} n, \varepsilon^{-1} s\right)=n-\varepsilon m(\Pi)-\varepsilon \widetilde{z}\left(\varepsilon^{-1} n, \varepsilon^{-1} s\right) .
$$

Consequently, formulas (28) lead to the identity

$$
\begin{aligned}
U_{\varepsilon}(x)= & \chi\left(\varepsilon^{-1} r\right)\left(u_{0}(x)-\varepsilon m(\Pi) u^{\prime}(x)\right)+\left(1-\chi\left(\varepsilon^{-1} r\right)\right)\left(\widehat{u}_{0}(x)-\varepsilon m(\Pi) \widehat{u}^{\prime}(x)\right) \\
& +\left(1-\chi\left(\varepsilon^{-1} r\right)\right) \chi^{0}(x) \partial_{n} u_{0}(0, s)\left(n-\varepsilon m(\Pi)-\varepsilon \widetilde{z}\left(\varepsilon^{-1} n, \varepsilon^{-1} s\right)\right) \\
= & \mathcal{U}_{\varepsilon}(x)+\mathcal{U}_{\varepsilon}^{1}(x)+\mathcal{U}_{\varepsilon}^{2}(x),
\end{aligned}
$$

where

$$
\begin{aligned}
& \mathcal{U}_{\varepsilon}^{1}(x)=\chi\left(\varepsilon^{-1} r\right)\left(u_{0}(x)-\varepsilon m(\Pi) u^{\prime}(x)\right) \\
& \mathcal{U}_{\varepsilon}^{2}(x)=\chi_{\varepsilon}^{1}(x)\left(\widehat{u}_{0}(x)-\varepsilon m(\Pi) \widehat{u}^{\prime}(x)\right)
\end{aligned}
$$


Recalling inequalities (22) and (26), we see that

$$
\begin{aligned}
\| \mathcal{U}_{\varepsilon}^{1} ; & H^{1}(\Omega(\varepsilon)) \|^{2} \\
\leq c \int_{\Omega \cap \mathbb{B}_{2 \varepsilon R}}\left(\varepsilon^{-2}\left|u_{0}(x)\right|^{2}+\left|u^{\prime}(x)\right|^{2}\right. & \left.+\left|\nabla_{x} u_{0}(x)\right|^{2}+\varepsilon^{2}\left|\nabla_{x} u^{\prime}(x)\right|^{2}\right) d x \\
\leq c \varepsilon^{6-2 \sigma-2} \int_{\Omega \cap \mathbb{B}_{2 \varepsilon R}}\left(r^{2(\sigma-3)}\left|u_{0}(x)\right|^{2}\right. & +r^{2(\sigma-2)}\left(\left|\nabla_{x} u_{0}(x)\right|^{2}+\left|u^{\prime}(x)\right|^{2}\right) \\
& \left.+r^{2(\sigma-1)}\left|\nabla_{x} u^{\prime}(x)\right|^{2}\right) d x \\
\leq c \varepsilon^{2(2-\sigma)}\left\|f ; V_{\sigma}^{1}(\Omega)\right\|^{2} . &
\end{aligned}
$$

We have used the fact that $\varepsilon^{-1} \leq c r^{-1}$ in the integration domain. By (24), (28), and (29), we have

$$
\begin{aligned}
& \left\|\mathcal{U}_{\varepsilon}^{2} ; H^{1}(\Omega(\varepsilon))\right\|^{2} \\
& \quad \leq c \int_{\left(\Omega \cap \mathcal{V}_{C \varepsilon}\right) \backslash \mathbb{B}_{\varepsilon R}}\left(\varepsilon^{-2}\left|\widehat{u}_{0}(x)\right|^{2}+\left|\widehat{u}^{\prime}(x)\right|^{2}+\left|\nabla_{x} \widehat{u}_{0}(x)\right|^{2}+\varepsilon^{2}\left|\nabla_{x} \widehat{u}^{\prime}(x)\right|^{2}\right) d x \\
& \quad \leq c \varepsilon^{3} \frac{1}{\varepsilon} \int_{\left(\Omega \cap \mathcal{V}_{C \varepsilon}\right) \backslash \mathbb{B}_{\varepsilon R}} r^{2(\sigma-1)}\left(r^{4} \varrho(x)^{-4}\left|\widehat{u}_{0}(x)\right|^{2}\right. \\
& \left.\quad+r^{2} \varrho(x)^{-2}\left(\left|\widehat{u}^{\prime}(x)\right|^{2}+\left|\nabla_{x} \widehat{u}_{0}(x)\right|^{2}\right)+\left|\nabla_{x} \widehat{u}^{\prime}(x)\right|^{2}\right) d x \\
& \quad \leq c \varepsilon^{3}\left\|f ; V_{\sigma}^{1}(\Omega)\right\|^{2} .
\end{aligned}
$$

We note that, by condition (21) on the weight index $\sigma$, the exponent in the auxiliary factor $r^{2(\sigma-1)}$ is positive.

In the integral identity that serves problem (2) (see [18]), we take the difference $\mathcal{R}_{\varepsilon}=$ $u_{\varepsilon}-\mathcal{U}_{\varepsilon}$ for the role of a test function and subtract the scalar product $\left(\nabla_{x} \mathcal{U}_{\varepsilon}, \nabla_{x} \mathcal{R}_{\varepsilon}\right)_{\Omega(\varepsilon)}$ from both sides. We transform the right-hand side of the resulting identity

$$
\left(\nabla_{x} \mathcal{R}_{\varepsilon}, \nabla_{x} \mathcal{R}_{\varepsilon}\right)_{\Omega(\varepsilon)}=\left(f, \mathcal{R}_{\varepsilon}\right)_{\Omega(\varepsilon)}-\left(\nabla_{x} \mathcal{U}_{\varepsilon}, \nabla_{x} \mathcal{R}_{\varepsilon}\right)_{\Omega(\varepsilon)}
$$

assuming that the function $\mathcal{R}_{\varepsilon}$ is extended by zero from $\Omega(\varepsilon)$ to the larger domain $\Omega$. Using (31), (30), and (33), we find

$$
\begin{aligned}
\left(f, \mathcal{R}_{\varepsilon}\right)_{\Omega(\varepsilon)}- & \left(\nabla_{x} \mathcal{U}_{\varepsilon}, \nabla_{x} \mathcal{R}_{\varepsilon}\right)_{\Omega(\varepsilon)} \\
= & \left(f, \mathcal{R}_{\varepsilon}\right)_{\Omega}-\left(\nabla_{x}\left(u_{0}-\varepsilon m(\Pi) u^{\prime}\right), \nabla_{x} \mathcal{R}_{\varepsilon}\right)_{\Omega}+\left(\nabla_{x} \mathcal{U}_{\varepsilon}^{1}, \nabla_{x} \mathcal{R}_{\varepsilon}\right)_{\Omega(\varepsilon)} \\
& +\left(\nabla_{x} \mathcal{U}_{\varepsilon}^{2}, \nabla_{x} \mathcal{R}_{\varepsilon}\right)_{\Omega(\varepsilon)}+\varepsilon\left(\nabla_{x}(1-\chi) \chi^{0} \partial_{n} u_{0} \widetilde{z}, \nabla_{x} \mathcal{R}_{\varepsilon}\right)_{\Omega(\varepsilon)} .
\end{aligned}
$$

The first two terms on the right cancel, due to relations (7), (25) and the possibility to integrate by parts, because $\mathcal{R}_{\varepsilon}=0$ on $\partial \Omega$ and near the corner point $\mathcal{O}$, in view of the conditions introduced in Section 1. By (34) and (35), the moduli of the third and the fourth terms do not exceed the quantity

$$
c \varepsilon^{\min \{2-\sigma, 3 / 2\}}\left\|f ; V_{\sigma}^{1}(\Omega)\right\|\left\|\mathcal{R}_{\varepsilon} ; H^{1}(\Omega(\varepsilon))\right\| .
$$

The last term in (37) can be written as

$$
\begin{aligned}
& \varepsilon\left(\chi^{0} \widetilde{z} \nabla_{x}(1-\chi) \partial_{n} u_{0}, \nabla_{x} \mathcal{R}_{\varepsilon}\right)_{\Omega(\varepsilon)} \\
& \quad-\varepsilon\left(\nabla_{x} \chi^{0} \widetilde{z}, \mathcal{R}_{\varepsilon} \nabla_{x}(1-\chi) \partial_{n} u^{0}\right)_{\Omega(\varepsilon)}-\varepsilon\left((1-\chi) \partial_{n} u_{0} \Delta_{x} \chi^{0} \widetilde{z}, \mathcal{R}_{\varepsilon}\right)_{\Omega(\varepsilon)},
\end{aligned}
$$


and its modulus can be estimated by the same quantity (38) if we use (6), (22), (24), and the inequalities

$$
\begin{gathered}
\int_{\partial \Omega} r^{2 \sigma-3}\left(\left|\partial_{n} u_{0}(0, s)\right|^{2}+r^{2}\left|\partial_{s} \partial_{n} u_{0}(0, s)\right|^{2}\right) d s \leq c\left\|u_{0} ; V_{\sigma}^{3}(\Omega)\right\|^{2}, \\
\left\|r^{-1} \varrho^{-1} \mathcal{R}_{\varepsilon} ; L_{2}(\Omega(\varepsilon))\right\| \leq c\left\|\nabla_{x} \mathcal{R}_{\varepsilon} ; L_{2}(\Omega(\varepsilon))\right\|, \\
\int_{0} n^{2 j} e^{-2 n / \varepsilon} d n \leq c \varepsilon^{2 j+1}, \quad j=0,1 .
\end{gathered}
$$

The first of these estimates is the well-known trace inequality for Kondrat'ev spaces [24], and the second is a consequence of the 1-dimensional Hardy inequality (27). Also, when treating the last scalar product in (39), one should use the harmonicity of the function $\widetilde{z}$ and the representation of the Laplace operator in curvilinear coordinates,

$$
\Delta_{x}=A(n, s)^{-1}\left(\frac{\partial}{\partial n} A(n, s) \frac{\partial}{\partial n}+\frac{\partial}{\partial s} A(n, s)^{-1} \frac{\partial}{\partial s}\right)
$$

here $A(n, s)=1-n k(s)$ and $k$ is the curvature of the $\operatorname{arc} \Gamma \backslash \mathcal{O}$. In detail, the deduction of the estimate in question will be presented, for a somewhat more general situation, in the next section within the proof of Theorem 3.

The Friedrichs inequality

$$
\left\|\mathcal{R}_{\varepsilon} ; H^{1}(\Omega(\varepsilon))\right\| \leq c\left\|\nabla_{x} \mathcal{R}_{\varepsilon} ; L_{2}(\Omega(\varepsilon))\right\|
$$

Lemma 3, and the relations already proved for the terms in (37) establish the following statement.

Theorem 2. Assume that $0<\alpha<\pi$ and that relations (21) are fulfilled. Then the solution $u_{\varepsilon}$ of problem (2) and its asymptotic approximation (30), extended by zero to the domain $\Omega$, satisfy the inequality

$$
\left\|u_{\varepsilon}-U_{\varepsilon} ; H^{1}(\Omega(\varepsilon))\right\| \leq c_{\sigma} \varepsilon^{\min \{2-\sigma, 3 / 2\}}\left\|f ; V_{\sigma}^{1}(\Omega)\right\|,
$$

with $c_{\sigma}$ independent of $f$ and of the parameter $\varepsilon \in\left(0, \varepsilon_{0}\right]$.

It is not hard to check that for $\sigma<1$ the $H^{1}(\Omega(\varepsilon)$ )-norm of each term in the asymptotic approximation (30) is of lower order than the majorant in estimate (42), which means that, indeed, Theorem 2 justifies the asymptotics constructed.

\section{§5. The leading asymptotic terms in the Case Where $\alpha \in(\pi, 2 \pi]$}

If we deal with an incoming angle, then, since the gradient of the function $u_{0}$ is not square integrable (see (8)), the boundary-value problem (25) does not admit any energy solution $u^{\prime} \in H^{1}(\Omega)$. Under the modified conditions (21) (for $\alpha>\pi$ the semiinterval indicated before for the weight index $\sigma$ is empty), the existence of a solution $u^{\prime} \in V_{2}^{2}(\Omega) \subset L_{2}(\Omega)$ will be established, but in this class a solution is not unique, taking the form

$$
u^{\prime}=u_{0}^{\prime}+a \zeta
$$

where $u_{0}^{\prime}$ is a particular solution, $\zeta \in V_{2}^{2}(\Omega)$ is the weight function (10), and $a$ is an arbitrary constant. Our next goal is to find this constant.

We apply the method of matched expansions (see, e.g., the books $[16,17]$ ). The expansion

$$
u_{\varepsilon}(x) \approx u_{0}(x)-\varepsilon m(\Pi) u_{0}^{\prime}(x)+a_{0}(\varepsilon) \zeta(x)-\varepsilon \chi^{0}(x) \partial u_{0}(0, s) \widetilde{z}\left(\varepsilon^{-1} n, \varepsilon^{-1} s\right),
$$

viewed in the preceding section as global, now becomes external, i.e., it is accepted far from the corner point. The internal expansion is valid in the vicinity of $\mathcal{O}$ in accordance with the definition of the singularly perturbed domain $\Omega(\varepsilon)$, as given in Section 1; it is 
described in terms of solutions of problem (11) on an unbounded set. The leading term of the internal expansion must solve the homogeneous problem and possess an asymptotics at infinity that starts with the term

$$
K r^{\pi / \alpha} \cos (\pi \varphi / \alpha)=\varepsilon^{\pi / \alpha} K \rho^{\pi / \alpha} \cos (\pi \varphi / \alpha),
$$

which is prescribed by the representation (8) of the leading term of the external expansion (44). Therefore, in agreement with formula (15), we put

$$
u_{\varepsilon}(x) \approx \varepsilon^{\pi / \alpha} K Y\left(\varepsilon^{-1} x\right) .
$$

We continue matching the outer and inner expansions. For this, in the lower terms (16) of the function $Y$ we pass from the dilated coordinates $\xi$ to the usual coordinates $\chi$. First, let $\alpha \in(\pi, 2 \pi)$; then, by the definition (17), we have

$$
\begin{aligned}
\varepsilon^{\pi / \alpha} & K Y\left(\varepsilon^{-1} x\right) \\
= & K r^{\pi / \alpha} \cos \left(\frac{\pi}{\alpha} \varphi\right)+\varepsilon r^{-1+\pi / \alpha} K m(\Pi) \frac{\pi}{\alpha}\left[\cos \left(\frac{1}{2}(\alpha-\pi)\right)\right]^{-1}\left[\cos \left(1-\frac{\pi}{\alpha}\right) \varphi\right] \\
& -\varepsilon^{2 \pi / \alpha} K m(\Xi) r^{-\pi / \alpha} \cos \left(\frac{\pi}{\alpha} \varphi\right)-\varepsilon \chi^{0}(x) K \frac{\pi}{\alpha} r^{-1+\pi / \alpha} \widetilde{z}\left(\varepsilon^{-1} n, \varepsilon^{-1} s\right)+\cdots
\end{aligned}
$$

Here the dots hide the lower terms immaterial in our formal asymptotic analysis, and the cut-off functions $\chi_{ \pm}(\varphi)$ are replaced with $\chi^{0}(x)$; this modification is possible because the function is exponentially small off angular neighborhoods of the rays $\left\{x: r>\varepsilon^{1 / 2}\right.$, $\varphi=\alpha / 2\}$, i.e., on the set where the cut-off functions in question differ from each other. Observing that, by (8), the expression $K \pi \alpha^{-1} r^{-1+\pi / \alpha}$ is the leading term of the asymptotics for the quantity $\partial_{n} u_{0}(0, s)$ near the corner point, we find, on the right-hand side in (46), a term of the boundary layer type in the external expansion, which yields the asymptotics as $r \rightarrow 0$ of the function $(n, s) \mapsto \varepsilon \partial_{n} u_{0}(0, s) \widetilde{z}(\xi)$. Thus, the corresponding components of the expansions (45) and (46) match automatically. The same can be said about the second terms on the right in (44) and (46). The reason is that below (see Lemma 4) we shall establish the asymptotic representation

$$
u_{0}^{\prime}(x)=-\chi(r) K \pi \alpha^{-1} Y_{1}(x)+\widetilde{u}_{0}^{\prime}(x)
$$

of the solution of problem (25). By the definition (17) of the function $Y_{1}$, the second term on the right in (46) is none other than the asymptotic term shown in (47) times $-\varepsilon m(\Pi)$ (in accordance with (44)).

So, only the term

$$
-\varepsilon^{2 \pi / \alpha} K m(\Xi) r^{-\pi / \alpha} \cos (\pi \varphi / \alpha)
$$

needs matching. The solution of the homogeneous problem (7) with the singularity indicated above occurs in relations (10), (9) and (43), (44), and the factor $a_{0}(\varepsilon)$ should be fixed as follows:

$$
a_{0}(\varepsilon)=-\varepsilon^{2 \pi / \alpha} \pi K m(\Xi)
$$

If $\alpha=2 \pi$ (the angle $\mathbb{K}$ is the plane with a semi-infinite cut), then the component (17) of the expansions (16) and (47) involves the logarithm. The coordinate change $\xi \mapsto x=\varepsilon \xi$ gives rise to $\log \varepsilon$, and the second term on the right in (46) turns into

$$
-\frac{\varepsilon}{2 \pi} m(\Pi) K\left[r^{-1 / 2}\left(\log r \cos \left(\frac{\varphi}{2}\right)-\varphi \sin \left(\frac{\varphi}{2}\right)\right)+\log \varepsilon r^{-1 / 2} \cos \left(\frac{\varphi}{2}\right)\right] .
$$

As a result, the formula for $a_{0}(\varepsilon)$ becomes

$$
a_{0}(\varepsilon)=-\varepsilon K\left(\pi m(\Xi)+\frac{1}{2} m(\Pi) \log \varepsilon\right) .
$$

This finishes the procedure of matching and the formal construction of the leading terms of the asymptotics for the solution. We now pass to justifying the asymptotics. 
Suppose that

$$
f \in V_{\sigma}^{1}(\Omega), \quad \sigma \in(2-2 \pi / \alpha, 2-\pi / \alpha) .
$$

At first glance, it seems that the restriction imposed in (50) on the weight index $\sigma$ is stronger than (21) in the case where $\alpha<\pi$. Actually, the requirement (50) admits indices exceeding 1 , while (21) does not. We shall return to discussing the right conditions on $\sigma$ in Remark 3.

The general results of [24], applied to the Dirichlet problems (7) and (25) (see also the introductory Chapter 2 in [27]), yield the following statement in the case of an incoming angle.

Lemma 4. Suppose $\alpha \in(\pi, 2 \pi]$, and assume conditions (50).

1) Problem (7) has a unique solution $u_{0} \in \stackrel{\circ}{H}^{1}(\Omega)$; this solution admits representation as in (8) with a remainder term $\widetilde{u}_{0} \in V_{\sigma}^{3}(\Omega)$ and satisfies the estimate

$$
|K|+\left\|\widetilde{u}_{0} ; V_{\sigma}^{3}(\Omega)\right\| \leq c\left\|f ; V_{\sigma}^{1}(\Omega)\right\| .
$$

2) Problem (25) has a solution $u^{\prime} \in L_{2}(\Omega)$, and every such solution has the form (43), where $a$ is a constant and $u_{0}^{\prime}$ is a particular solution representable in the form (47) with $Y_{1}$ as in (17), and the norm of $\widetilde{u}_{0}^{\prime} \in V_{\sigma}^{2}(\Omega)$ does not exceed the quantity $c\left\|f ; V_{\sigma}^{1}(\Omega)\right\|$.

It is easily seen that the function $\widetilde{u}_{0} \in V_{\sigma}^{3}(\Omega)$ solves problem $(7)$ with the right-hand side

$$
\left(x \mapsto f(x)-K\left[\Delta_{x}, \chi(x)\right] r^{\pi / \alpha} \cos (\pi \varphi / \alpha)\right) \in V_{\sigma}^{1}(\Omega) .
$$

Indeed, the subtrahend, which involves the commutator $\left[\Delta_{x}, \chi\right]$, belongs to $C_{c}^{\infty}(\bar{\Omega} \backslash \mathcal{O})$. Therefore, all the conclusions made in Section 4 about the solution $u_{0}$ remain valid for the remainder term $\widetilde{u}_{0}$ in the asymptotic representation (8). The same can be said about the trace $\partial_{n} \widetilde{u}_{0}(0, \cdot)$ and about $\widetilde{u}_{0}^{\prime}$ in $(47)$. Namely, the components in the expansions

$$
\widetilde{u}_{0}(x)=\chi^{0}(x) n \partial_{n} \widetilde{u}_{0}(0, s)+\widehat{\widetilde{u}}_{0}(x), \quad \widetilde{u}_{0}^{\prime}(x)=\chi^{0}(x) \partial_{n} \widetilde{u}_{0}(0, s)+\widehat{\widetilde{u}}_{0}^{\prime}(x)
$$

satisfy the inequalities

$$
\begin{aligned}
& \left\|r^{\sigma-1 / 2} \partial_{s} \partial_{n} \widetilde{u}_{0}(0, \cdot) ; L_{2}(\Gamma)\right\|+\left\|r^{\sigma-3 / 2} \partial_{n} \widetilde{u}_{0}(0, \cdot) ; L_{2}(\Gamma)\right\| \leq c\left\|f ; V_{\sigma}^{1}(\Omega)\right\|, \\
& \frac{1}{\delta} \int_{\Omega \cap \mathcal{V}_{\delta}} r^{2 \sigma-6}\left[\varrho(x)^{-4}\left|\widehat{\widetilde{u}}_{0}(x)\right|^{2}\right. \\
& \left.+r^{2} \varrho(x)^{-2}\left(\left|\widehat{\widetilde{u}}_{0}^{\prime}(x)\right|^{2}+\left|\nabla_{x} \widehat{\widetilde{u}}_{0}(x)\right|^{2}\right)+r^{4}\left|\nabla_{x} \widehat{\widetilde{u}}_{0}^{\prime}(x)\right|^{2}\right] d x \\
& \leq c\left\|f ; V_{\sigma}^{1}(\Omega)\right\|^{2}
\end{aligned}
$$

here the index $\sigma$ is subject to (50). Since the weight function $\zeta$ occurring in (10) and (9), is a solution of the homogeneous problem (7), it is infinitely differentiable everywhere except for the point $\mathcal{O}$. In particular, for its regular part $\zeta_{0}$ we have

$$
\left|\zeta_{0}(x)\right| \leq c_{0} r^{\pi / \alpha} \varrho(x), \quad\left|\nabla_{x} \zeta_{0}(x)\right| \leq c_{1} r^{-1+\pi / \alpha} .
$$

In order to "glue" the outer expansion (44) and the inner expansion (45), we apply the construction suggested in [28] (see also [13, §2.1] and [21, §4]). Namely, from the functions $u_{0}, u_{0}^{\prime}$, and $\zeta$, we split off the terms that prevent them from being as smooth as required, but are present in the expression $\varepsilon^{\pi / \alpha} K \chi(r) Y\left(\varepsilon^{-1} x\right)$, in accordance with formulas (15) and (16). We put

$$
\begin{aligned}
U_{\varepsilon}(x)= & \varepsilon^{\pi / \alpha} K \chi(r) Y\left(\varepsilon^{-1} x\right)+\widetilde{u}_{0}(x)-\varepsilon m(\Pi) \widetilde{u}_{0}^{\prime}(x)+a_{0}(\varepsilon) \zeta_{0}(x) \\
& -\varepsilon\left(1-\chi\left(\varepsilon^{-1} r\right)\right) \chi^{0}(x) \partial_{n} \widetilde{u}_{0}(0, s) \widetilde{z}\left(\varepsilon^{-1} n, \varepsilon^{-1} s\right)
\end{aligned}
$$


here $a_{0}(\varepsilon)$ is as in (48) or (49). We can write the approximate solution (55) in a different way: replacing the growing solution (15) of the homogeneous problem (11) with the decaying remainder $\widetilde{Y}$ (see the definition (16)) and recovering the terms that were split off from $u_{0}, u_{0}^{\prime}$, and $\zeta$, we obtain

$$
\begin{aligned}
U_{\varepsilon}(x)= & u_{0}(x)-\varepsilon m(\Pi) u_{0}^{\prime}(x)+a_{0}(\varepsilon) \zeta(x)+\varepsilon^{\pi / \alpha} K \chi(r) \widetilde{Y}\left(\varepsilon^{-1} x\right) \\
& -\varepsilon\left(1-\chi\left(\varepsilon^{-1} r\right)\right) \chi^{0}(x) \partial_{n} u_{0}(0, s) \widetilde{z}\left(\varepsilon^{-1} n, \varepsilon^{-1} s\right) .
\end{aligned}
$$

The first three terms on the right are solutions of the Dirichlet problem in $\Omega$, and the last two are correction terms of a boundary layer type, power-law near the corner point and exponential near the rapidly oscillating boundary, respectively. Contrary to formula (55), where $\widetilde{u}_{0}$ and $\widetilde{u}_{0}^{\prime}, \zeta_{0}$ belong to the Sobolev spaces $H^{2}(\Omega)$ and $H^{1}(\Omega)$, formula $(56)$ involves the terms $u_{0}^{\prime}$ and $\zeta$ that only fall into the Lebesgue space $L_{2}(\Omega)$. Nevertheless, expression (56) will be useful in Section 7, where we shall deal with selfadjoint extensions of the Dirichlet problem operator.

As in Section 4 , in addition to $U_{\varepsilon}$ we need an intermediate asymptotic approximation, which we obtain by the same rule as in the case of obtaining (31) from (30) (the term $\varepsilon^{\pi / \alpha} K \chi \widetilde{Y}$ remains intact):

$$
\begin{aligned}
\mathcal{U}_{\varepsilon}(x)= & \varepsilon^{\pi / \alpha} K \chi(r) \widetilde{Y} \\
& +\left(1-\chi\left(\varepsilon^{-1} r\right)\right)\left[( 1 - \chi _ { \varepsilon } ^ { 1 } ( x ) ) \left(\widehat{\widetilde{u}}_{0}(x)\right.\right. \\
& \left.-\varepsilon m(\Pi) \widehat{\widetilde{u}}^{\prime}(x)+a_{0}(\varepsilon) \zeta_{0}(x)\right) \\
& \left.+\varepsilon \chi^{0}(x) \partial_{n} \widetilde{u}_{0}(0, s) z\left(\varepsilon^{-1} n, \varepsilon^{-1} s\right)\right] .
\end{aligned}
$$

Remark 3. Since the first terms on the right in (55) and (57) coincide, and $\zeta_{0}$ admits estimate (54), it is not hard to show that the difference $\mathcal{U}_{\varepsilon}-U_{\varepsilon}$ satisfies an inequality similar to $(32)$ with the majorant

$$
c\left(\varepsilon^{\max \{2-\sigma, 3 / 2\}}+\varepsilon^{2 \pi / \alpha+1 / 2}\left(1+\delta_{\alpha, 2 \pi}|\log \varepsilon|\right)\right)\left\|f ; V_{\sigma}^{1}(\Omega)\right\| .
$$

Unlike the case of $\alpha \in(0, \pi)$, treated in Lemma 3, the inequality mentioned above is less than satisfactory. We explain the reason. The restriction (21) allows us to fix the weight index

$$
\sigma= \begin{cases}1 / 2 & \text { if } \alpha<2 \pi / 3, \\ \delta+2-\pi / \alpha & \text { if } \alpha \in[2 \pi / 3, \pi)\end{cases}
$$

where $\delta$ is an arbitrarily small positive number. In this case the exponent of $\varepsilon$ on the right in (32) and (42) becomes

$$
\begin{cases}3 / 2 & \text { if } \alpha<2 \pi / 3, \\ -\delta+\pi / \alpha & \text { if } \alpha \in[2 \pi / 3, \pi) .\end{cases}
$$

Therefore, for all angles $\alpha<\pi$ we can make this exponent larger than 1 , thus justifying the presence of each term in the asymptotic approximation (30) to the solution of problem (2). It should be noted that no right-hand side of (42) with a factor of $o\left(\varepsilon^{3 / 2}\right)$ is possible, because the asymptotics does not include the next term $\varepsilon^{2} \chi^{0}(x) z^{1}\left(\varepsilon^{-1} n, \varepsilon^{-1} s\right)$ of boundary layer type, the norm of which in the space $H^{1}\left(\Omega \backslash \mathbb{B}_{R^{0} / 2}\right)$ is $O\left(\varepsilon^{3 / 2}\right)$.

For $\alpha \in(\pi, 2 \pi]$, the restriction (50) implies that the minimal exponent of $\varepsilon$ in the majorant (58) is equal to

$$
\begin{cases}3 / 2 & \text { if } \alpha<4 \pi / 3, \\ -\delta+2 \pi / \alpha & \text { if } \alpha \in[4 \pi / 3,2 \pi],\end{cases}
$$

where $\delta>0$ is arbitrary, as before. Consequently, for no angle $\alpha \in(\pi, 2 \pi]$ can we justify inclusion of the term $a_{0}(\varepsilon) \zeta_{0}$ or $a_{0}(\varepsilon) \zeta$ in the asymptotic approximation (55) or (56) to 
the solution $u_{\varepsilon}$ of problem (2) (cf. relations (48) and (49)). In the next two statements we shall show that accuracy estimates for approximation in the norm $\left\|\cdot ; V_{\varkappa}^{1}(\Omega(\varepsilon))\right\|$, as defined in (20), make it possible to justify the presence of the terms mentioned above in the global asymptotic approximation $U_{\varepsilon}$. Passage to weighted norms preserves the possibility to apply, in Section 7, the asymptotic representation constructed for modeling the problem in a domain with rapidly oscillating boundary.

Lemma 5. Let $\varkappa \geq 0$, and let conditions (50) be fulfilled. Then the functions (55) and (57) satisfy the inequality

$$
\left\|\mathcal{U}_{\varepsilon}-U_{\varepsilon} ; V_{\varkappa}^{1}(\Omega(\varepsilon))\right\| \leq c\left(\varepsilon^{\varkappa+2-\sigma}+\varepsilon^{3 / 2}\left(1+\delta_{\alpha, 2 \pi}|\log \varepsilon|\right)\right)\left\|f ; V_{\sigma}^{1}(\Omega)\right\|,
$$

where $c$ is a constant independent of the parameter $\varepsilon \in\left(0, \varepsilon_{0}\right]$ and of the right-hand side $f$ of problem (7).

Proof. By the definitions (55) and (57), we have

$$
U_{\varepsilon}(x)-\mathcal{U}_{\varepsilon}(x)=\mathcal{U}_{\varepsilon}^{1}(x)+\mathcal{U}_{\varepsilon}^{2}(x)-\mathcal{U}_{\varepsilon}^{3}(x)
$$

here $\mathcal{U}_{\varepsilon}^{1}$ and $\mathcal{U}_{\varepsilon}^{2}$ are the expressions (33) in which the quantities $u_{0}, \widetilde{u}_{0}$ and $u^{\prime}, \widetilde{u}^{\prime}$ are replaced, respectively, with the terms $\widetilde{u}_{0}, \widehat{\widetilde{u}}^{\prime}$ and $\widetilde{u}_{0}^{\prime}, \widehat{\widetilde{u}}_{0}^{\prime}$ of the expansions (52), and

$$
\mathcal{U}_{\varepsilon}^{3}(x)=a_{0}(\varepsilon)\left(\chi(r)+\left(1-\chi\left(\varepsilon^{-1} r\right)\right) \chi_{\varepsilon}^{1}(x)\right) \zeta_{0}(x) .
$$

Using (54), (51) and (48), (49), we obtain

$$
\begin{aligned}
\left\|\mathcal{U}_{\varepsilon}^{3} ; V_{\varkappa}^{1}(\Omega)\right\| & \leq c \varepsilon^{2 \pi / \alpha}\left(1+\delta_{\alpha, 2 \pi}|\log \varepsilon|\right)|K|\left(\int_{0}^{\varepsilon R^{0}} r^{2(\varkappa+\pi / \alpha)}\left(\varepsilon^{-2}+r^{-2}\right) r d r\right. \\
& \left.+r^{2(\varkappa+\pi / \alpha)} \int_{0}^{d r \varepsilon}\left(\varrho(x)^{-2} \varepsilon^{-2}+r^{-2}\right) d n d s\right)^{1 / 2} \\
& \leq c \varepsilon^{2 \pi / \alpha}\left(1+\delta_{\alpha, 2 \pi}|\log \varepsilon|\right)\left\|f ; V_{\sigma}^{1}(\Omega)\right\| .
\end{aligned}
$$

Note that for $\varkappa=0$ this chain of inequalities ensures the majorant (58) indicated above.

Estimation of $\left\|\mathcal{U}_{\varepsilon}^{2} ; V_{\varkappa}^{1}(\Omega)\right\|$ repeats the calculation (35); the presence of the additional factor $r^{2 \varkappa}$, which is small near $\mathcal{O}$, changes nothing. On the other hand, (34) modifies as follows (we use Lemma 4):

$$
\begin{aligned}
& \left\|\mathcal{U}_{\varepsilon}^{1} ; V_{\varkappa}^{1}(\Omega)\right\|^{2} \\
& \leq c \int_{\Omega \cap \mathbb{B}_{2 R \varepsilon}} r^{2 \varkappa}\left[\left(\varepsilon^{-2}+r^{-2}\right)\left|\widehat{\widetilde{u}}_{0}(x)\right|^{2}\right. \\
& \left.+\left(1+\varepsilon^{2} r^{-2}\right)\left(\left|\widehat{\widetilde{u}}_{0}^{\prime}(x)\right|^{2}+\left|\nabla_{x} \widehat{\widetilde{u}}_{0}(x)\right|^{2}\right)+\varepsilon^{2}\left|\nabla_{x} \widehat{\widetilde{u}}_{0}^{\prime}(x)\right|^{2}\right] d x \\
& \leq c \varepsilon^{2 \varkappa+4-2 \sigma} \int_{\Omega \cap \mathbb{B}_{2 R \varepsilon}} r^{2 \sigma-6}\left[\left|\widehat{\widetilde{u}}_{0}(x)\right|^{2}+r^{2}\left(\left|\widehat{\widetilde{u}}_{0}^{\prime}(x)\right|^{2}+\left|\nabla_{x} \widehat{\widetilde{u}}_{0}(x)\right|^{2}\right)+r^{4}\left|\nabla_{x} \widehat{\widetilde{u}}_{0}^{\prime}(x)\right|^{2}\right] d x \\
& \leq c \varepsilon^{2(\varkappa+2-\sigma)}\left\|f ; V_{\sigma}^{1}(\Omega)\right\|^{2} \text {. }
\end{aligned}
$$

Lemma 6. Suppose that $\varkappa \in(-\pi / \alpha, \pi / \alpha)$ and $f \in L_{2}(\Omega(\varepsilon))$. Then the solution $u_{\varepsilon} \in$ $H^{2}(\Omega(\varepsilon)) \cap \stackrel{\circ}{H}^{1}(\Omega(\varepsilon))$ of problem (2) satisfies the estimate

$$
\left\|u_{\varepsilon} ; V_{\varkappa}^{1}(\Omega(\varepsilon))\right\| \leq c\left\|r^{\varkappa+1} f ; L_{2}(\Omega(\varepsilon))\right\|=c\left\|f ; V_{1+\varkappa}^{0}(\Omega(\varepsilon))\right\|
$$

in which the norm $\left\|\cdot ; V_{\varkappa}^{1}(\Omega(\varepsilon))\right\|$ is defined by a formula similar to (20), and $c_{\varkappa}$ is a constant independent of $f$ and of the parameter $\varepsilon \in\left(0, \varepsilon_{0}\right]$. 
Proof. Since the boundary $\partial \Omega(\varepsilon)$ is smooth and does not contain $\mathcal{O}$, the solution $u_{\varepsilon} \in$ $H^{2}(\Omega(\varepsilon))$ exists and is unique, and the function spaces $L_{2}(\Omega(\varepsilon))$ and $V_{\varkappa+1}^{0}(\Omega(\varepsilon))$ coincide algebraically and topologically.

We introduce the following weight function, continuous in $\bar{\Omega} \backslash \mathcal{O}$ :

$$
\mathcal{T}_{t}(x)= \begin{cases}r^{\varkappa} & \text { if } r \geq t \\ t^{\varkappa} & \text { if } r \leq t\end{cases}
$$

where $t$ is a positive number. We plug the test function $\mathcal{T}_{t}^{2} u_{\varepsilon}=\mathcal{T}_{t} \mathbf{u}_{\varepsilon}$ into the integral identity (see [18]) that serves problem (2); this test function belongs to the space $\stackrel{\circ}{H}^{1}(\Omega(\varepsilon))$ because, obviously,

$$
\left|\nabla_{x} \mathcal{T}_{t}(x)\right| \leq c|\varkappa| r^{\varkappa-1} \text { for } r>t, \quad \nabla_{x} \mathcal{T}_{t}(x)=0 \text { for } r \leq t
$$

After easy computations, we get

$$
\begin{aligned}
& \left(\mathcal{T}_{t} f, \mathbf{u}_{\varepsilon}\right)_{\Omega(\varepsilon)}=\left(f, \mathcal{T}_{t}^{2} u_{\varepsilon}\right)_{\Omega(\varepsilon)} \\
& \quad=\left(\nabla_{x} u_{\varepsilon}, \nabla_{x} \mathcal{T}_{t}^{2} \mathbf{u}_{\varepsilon}\right)_{\Omega(\varepsilon)}=\left(\mathcal{T}_{t} \nabla_{x} u_{\varepsilon}, \nabla_{x} \mathbf{u}_{\varepsilon}+\mathcal{T}_{t}^{-1} \mathbf{u}_{\varepsilon} \nabla_{x} \mathcal{T}_{t}\right)_{\Omega(\varepsilon)} \\
& \quad=\left(\nabla_{x} \mathbf{u}_{\varepsilon}, \nabla_{x} \mathbf{u}_{\varepsilon}+\mathcal{T}_{t}^{-1} \mathbf{u}_{\varepsilon} \nabla_{x} \mathcal{T}_{t}\right)_{\Omega(\varepsilon)}-\left(\mathcal{T}_{t}^{-1} \mathbf{u}_{\varepsilon} \nabla_{x} \mathcal{T}_{t}, \nabla_{x} \mathbf{u}_{\varepsilon}+\mathcal{T}_{t}^{-1} \mathbf{u}_{\varepsilon} \nabla_{x} \mathcal{T}_{t}\right)_{\Omega(\varepsilon)} \\
& \quad=\left\|\nabla_{x} \mathbf{u}_{\varepsilon} ; L_{2}(\Omega(\varepsilon))\right\|^{2}-|\varkappa|^{2}\left\|r^{-1} \mathbf{u}_{\varepsilon} ; L_{2}\left(\Omega(\varepsilon) \backslash \mathbb{B}_{t}\right)\right\|^{2}
\end{aligned}
$$

We extend the function $\mathbf{u}_{\varepsilon} \in \stackrel{\circ}{H}^{1}(\Omega(\varepsilon))$ by zero to $\mathbb{K} \backslash \Omega(\varepsilon)$ and observe that, by the 1-dimensional Friedrichs inequality

$$
\int_{-\alpha / 2}^{\alpha / 2}\left|\frac{d \mathbf{U}}{d \varphi}(\varphi)\right|^{2} d \varphi \geq \frac{\pi^{2}}{\alpha^{2}} \int_{-\alpha / 2}^{\alpha / 2}|\mathbf{U}(\varphi)|^{2} d \varphi, \quad \mathbf{U} \in C_{0}^{1}\left(-\frac{\alpha}{2}, \frac{\alpha}{2}\right),
$$

we have

$$
\left\|\nabla_{x} \mathbf{u}_{\varepsilon} ; L_{2}(\Omega)\right\|^{2} \geq \pi^{2} \alpha^{-2}\left\|r^{-1} \mathbf{u}_{\varepsilon} ; L_{2}(\Omega)\right\|^{2}
$$

Consequently,

$$
\left\|\nabla_{x} \mathbf{u}_{\varepsilon} ; L_{2}(\Omega(\varepsilon))\right\|^{2}+\left\|r^{-1} \mathbf{u}_{\varepsilon} ; L_{2}(\Omega(\varepsilon))\right\|^{2} \leq c_{\varkappa}\left\|r \mathcal{T}_{t} f ; L_{2}(\varepsilon)\right\|^{2},
$$

and the claim follows by the passage to the limit as $t \rightarrow+0$.

We remark that the factor $c_{\varkappa}$ on the right in (61) grows unboundedly as $\varkappa \rightarrow \pm \pi / \alpha$, but we will not check this.

Now everything is ready for the proof of the main theorem pertaining to asymptotics in the case where $\alpha \in(\pi, 2 \pi]$.

Theorem 3. Suppose $\alpha \in(\pi, 2 \pi], \varkappa \in[0, \pi / \alpha)$, and conditions (50) are fulfilled. Then the solution $u_{\varepsilon} \in H^{2}(\Omega(\varepsilon)) \cap \stackrel{\circ}{H^{1}}(\Omega(\varepsilon))$ of problem (2) and its asymptotic approximation $(55)=(56)$ satisfy the inequality

$$
\left\|u_{\varepsilon}-U_{\varepsilon} ; V_{\varkappa}^{1}(\Omega(\varepsilon))\right\| \leq c_{\sigma, \varkappa}\left(\varepsilon^{\varkappa+2-\sigma}+\varepsilon^{3 / 2}\left(1+\delta_{\alpha, 2 \pi}|\log \varepsilon|\right)\right)\left\|f ; V_{\sigma}^{1}(\Omega)\right\|,
$$

with a constant $c_{\sigma, \varkappa}$ depending on the weight indices $\sigma$ and $\varkappa$, but independent of the parameter $\varepsilon \in\left(0, \varepsilon_{0}\right]$ and of the function $f$.

Proof. The procedure of checking inequality (64) remains largely the same as at the end of Section 4, but now formula (36) should be replaced with the formula

$$
\begin{gathered}
\left(\nabla_{x} \mathbf{R}_{\varepsilon}, \nabla_{x} \mathbf{R}_{\varepsilon}\right)_{\Omega(\varepsilon)}-\left(r^{-\varkappa} \mathbf{R}_{\varepsilon} \nabla_{x} r^{\varkappa}, r^{-\varkappa} \mathbf{R}_{\varepsilon} \nabla_{x} r^{\varkappa}\right)_{\Omega(\varepsilon)} \\
=\left(r^{\varkappa} f, \mathbf{R}_{\varepsilon}\right)_{\Omega(\varepsilon)}-\left(\nabla_{x} \mathcal{U}_{\varepsilon}, \nabla_{x} r^{\varkappa} \mathbf{R}_{\varepsilon}\right)_{\Omega(\varepsilon)},
\end{gathered}
$$

which involves the expression $\mathbf{R}_{\varepsilon}=r^{\varkappa}\left(u_{\varepsilon}-\mathcal{U}_{\varepsilon}\right)$. To obtain identity (65), it suffices to take the product $r^{2 \varkappa}\left(u_{\varepsilon}-\mathcal{U}_{\varepsilon}\right)=r^{\varkappa} \mathbf{R}_{\varepsilon}$ (which belongs to $\stackrel{\circ}{H}^{1}(\Omega(\varepsilon)$ ), as before, since $\mathcal{O} \notin \bar{\Omega}$ ) 
for the role of the test function in the integral identity, and to perform transformations similar to (62). Inequality (63) shows that the left-hand side of (65) is larger than the quantity

$$
C_{\varkappa}\left\|\nabla_{x} \mathbf{R}_{\varepsilon} ; V_{0}^{1}(\Omega(\varepsilon))\right\| \geq c_{\varkappa}\left\|u_{\varepsilon}-\mathcal{U}_{\varepsilon} ; V_{\varkappa}^{1}(\Omega(\varepsilon))\right\|
$$

here $C_{\varkappa}$ and $c_{\varkappa}$ are positive constants.

So, it remains to estimate the expression on the right in (65). Using (60) and (59), we obtain

$$
\begin{aligned}
& \left|\left(\nabla_{x} \mathcal{U}_{\varepsilon}, \nabla_{x} r^{\varkappa} \mathbf{R}_{\varepsilon}\right)_{\Omega(\varepsilon)}-\left(\nabla_{x} U_{\varepsilon}, \nabla_{x} r^{\varkappa} \mathbf{R}_{\varepsilon}\right)_{\Omega(\varepsilon)}\right| \\
& \quad \leq c \sum_{q=1}^{3}\left\|\mathcal{U}_{\varepsilon}^{q} ; V_{\varkappa}^{1}(\Omega(\varepsilon))\right\|\left(\left\|\nabla_{x} \mathbf{R}_{\varepsilon} ; L_{2}(\Omega(\varepsilon))\right\|+\left\|r^{-1} \mathbf{R}_{\varepsilon} ; L_{2}(\Omega(\varepsilon))\right\|\right) \\
& \quad \leq c\left(\varepsilon^{\varkappa+2-\sigma}+\varepsilon^{3 / 2}\left(1+\delta_{\alpha, 2 \pi}|\log \varepsilon|\right)\right)\left\|f ; V_{\sigma}^{1}(\Omega)\right\|\left\|\mathbf{R}_{\varepsilon} ; H^{1}(\Omega(\varepsilon))\right\| .
\end{aligned}
$$

We use the representation (55) of the approximate solution $U_{\varepsilon}$. We have

$$
\begin{aligned}
\left(\nabla_{x} U_{\varepsilon},\right. & \left.\nabla_{x} r^{\varkappa} \mathbf{R}_{\varepsilon}\right)_{\Omega(\varepsilon)} \\
= & \left(\nabla_{x}\left(u_{0}-\chi K r^{\pi / \alpha} \cos (\pi \varphi / \alpha)\right), \nabla_{x} r^{\varkappa} \mathbf{R}_{\varepsilon}\right)_{\Omega(\varepsilon)} \\
& -\varepsilon m(\Pi)\left(\nabla_{x}\left(u_{0}^{\prime}-\chi K \pi^{-1} \alpha Y_{1}\right), \nabla_{x} r^{\varkappa} \mathbf{R}_{\varepsilon}\right)_{\Omega(\varepsilon)} \\
& +a_{0}(\varepsilon)\left(\left(\zeta-\chi \pi^{-1} r^{-\pi / \alpha} \cos (\pi \varphi / \alpha)\right), \nabla_{x} r^{\varkappa} \mathbf{R}\right)_{\Omega(\varepsilon)} \\
& -\varepsilon\left(\nabla_{x} \chi \widetilde{z}^{0}(1-\chi) \partial_{n} \widetilde{u}_{0}, \nabla_{x} r^{\varkappa} \mathbf{R}_{\varepsilon}\right)_{\Omega(\varepsilon)}+K \varepsilon^{\pi / \alpha}\left(\nabla_{x} \chi Y, \nabla_{x} r^{\varkappa} \mathbf{R}_{\varepsilon}\right)_{\Omega(\varepsilon)} .
\end{aligned}
$$

First, we rewrite the last term, using formulas (15)-(17) for the solution $Y$ of the homogeneous problem (11):

$$
\begin{aligned}
K \varepsilon^{\pi / \alpha} & \left(\nabla_{x} \chi Y, \nabla_{x} r^{\varkappa} \mathbf{R}_{\varepsilon}\right)_{\Omega(\varepsilon)} \\
= & K \varepsilon^{\pi / \alpha}\left(\Delta_{x} \chi Y, r^{\varkappa} \mathbf{R}_{\varepsilon}\right)_{\Omega(\varepsilon)}=K \varepsilon^{\pi / \alpha}\left(\left[\Delta_{x}, \chi\right] Y, r^{\varkappa} \mathbf{R}_{\varepsilon}\right)_{\Omega(\varepsilon)} \\
= & K \varepsilon^{\pi / \alpha}\left(\left[\Delta_{x}, \chi\right] \widetilde{Y}, \nabla_{x} r^{\varkappa} \mathbf{R}_{\varepsilon}\right)_{\Omega(\varepsilon)}-K \varepsilon \pi \alpha^{-1}\left(\left[\Delta_{x}, \chi\right] r^{-1+\pi / \alpha} \chi 0 \widetilde{z}, r^{\varkappa} \mathbf{R}_{\varepsilon}\right)_{\Omega(\varepsilon)} \\
& +\left([ \Delta _ { x } , \chi ] \left(K r^{\pi / \alpha} \cos (\pi \varphi / \alpha)-\varepsilon m(\Pi) K \pi^{-1} \alpha Y_{1}\right.\right. \\
& \left.\left.+a_{0} \pi^{-1} r^{-\pi / \alpha} \cos (\pi \varphi / \alpha)\right), r^{\varkappa} \mathbf{R}\right)_{\Omega(\varepsilon)} .
\end{aligned}
$$

After easy computations, which take into account the harmonicity of the terms that were split off, we can distribute the components of the third scalar product on the right in (67) among the first three terms on the right in (66). We find that the corresponding sum is equal to

$$
\begin{aligned}
& \left(\nabla_{x}\left(u_{0}-\varepsilon m(\Pi) u_{0}^{\prime}+a_{0}(\varepsilon) \zeta\right), \nabla_{x} r^{\varkappa} \mathbf{R}\right)_{\Omega(\varepsilon)} \\
& \quad=-\left(\Delta_{x}\left(u_{0}-\varepsilon m(\Pi) u_{0}^{\prime}+a_{0}(\varepsilon) \zeta\right), r^{\varkappa} \mathbf{R}\right)_{\Omega}=\left(f, r^{\varkappa} \mathbf{R}\right)_{\Omega}
\end{aligned}
$$

and, thus, cancels in formula (65). It should be noted that the possibility to integrate by parts in the middle part of (65) is ensured by the fact that we can extend $\mathbf{R}_{\varepsilon}$ by zero to an $H^{1}$-function in the domain $\Omega$, and $\mathbf{R}_{\varepsilon}=0$ in a small neighborhood of $\mathcal{O}$. 
The first and second terms on the right in (66) can be handled as follows:

$$
\begin{aligned}
|K| \varepsilon^{\pi / \alpha}\left|\left(\left[\Delta_{\varkappa}, \chi\right] \widetilde{Y}, r^{\varkappa} \mathbf{R}_{\varepsilon}\right)_{\Omega(\varepsilon)}\right| & \\
\leq & c \varepsilon^{\pi / \alpha}\left\|f ; V_{\sigma}^{1}(\Omega)\right\|\left(\int_{\Omega \cap\left(\mathbb{B}_{\left.R^{0} \backslash \mathbb{B}_{R^{0} / 2}\right)}\right.}\left(\left|\widetilde{Y}\left(\varepsilon^{-1} x\right)\right|^{2}+\left|\nabla_{x} \widetilde{Y}\left(\varepsilon^{-1} x\right)\right|^{2}\right) d x\right)^{1 / 2} \\
& \times\left\|\mathbf{R}_{\varepsilon} ; L_{2}(\Omega(\varepsilon))\right\| \\
\leq & c \varepsilon^{\pi / \alpha}\left(\varepsilon^{\max \{2-\pi / \alpha, 2 \pi / \alpha\}}+\varepsilon^{1-\pi / \alpha}\left(\varepsilon+\varepsilon^{1 / 2}\right)\right)\left\|f ; V_{\sigma}^{1}(\Omega)\right\|\left\|\mathbf{R}_{\varepsilon} ; L_{2}(\Omega(\varepsilon))\right\| \\
\leq & c \varepsilon^{3 / 2}\left\|f ; V_{\sigma}^{1}(\Omega)\right\|\left\|\mathbf{R}_{\varepsilon} ; H^{1}(\Omega(\varepsilon))\right\|, \\
K \varepsilon\left|\left(\left[\Delta_{x}, \chi\right] r^{-1+\pi / \alpha} \chi \widetilde{z}^{0}, r^{\varkappa} \mathbf{R}_{\varepsilon}\right)_{\Omega(\varepsilon)}\right| & \\
\leq & c \varepsilon|K|\left(\int_{0}^{\infty}\left(1+\varepsilon^{-2} n^{2}\right) e^{-2 n / \varepsilon} d n\right)^{1 / 2}\left\|\mathbf{R}_{\varepsilon} ; L_{2}(\Omega(\varepsilon))\right\| \\
\leq & c \varepsilon^{3 / 2}\left\|f ; V_{\sigma}^{1}(\Omega)\right\|\left\|\mathbf{R}_{\varepsilon} ; H^{1}(\Omega(\varepsilon))\right\| .
\end{aligned}
$$

We have used inequalities (18), (19) and (6), (51), respectively.

It remains to consider the next to the last term in (66). As in (39), we obtain

$$
\begin{aligned}
& \varepsilon\left(\nabla_{x} \chi^{0} \widetilde{z}(1-\chi) \partial_{n} \widetilde{u}_{0}, \nabla_{x} r^{\varkappa} \mathbf{R}_{\varepsilon}\right)_{\Omega(\varepsilon)} \\
&=\varepsilon\left(\chi \widetilde{z} \nabla_{x}(1-\chi) \partial_{n} \widetilde{u}_{0}, \nabla_{x} r^{\varkappa} \mathbf{R}_{\varepsilon}\right)_{\Omega(\varepsilon)} \\
&-\varepsilon\left(\nabla_{x} \chi \widetilde{z}, r^{\varkappa} \mathbf{R}_{\varepsilon} \nabla_{x}(1-\chi) \partial_{n} \widetilde{u}_{0}\right)_{\Omega(\varepsilon)}-\varepsilon\left((1-\chi) \partial_{n} \widetilde{u}_{0} \Delta_{x} \chi^{0} \widetilde{z}, r^{\varkappa} \mathbf{R}_{\varepsilon}\right)_{\Omega(\varepsilon)} .
\end{aligned}
$$

The first two terms $I_{1}$ and $I_{2}$ on the right can be estimated with the help of (6), (40), and (53):

$$
\begin{aligned}
\left|I_{1}\right|+\left|I_{2}\right| & \leq c \varepsilon\left\|\mathbf{R}_{\varepsilon} ; H^{1}(\Omega(\varepsilon))\right\| \\
& \times\left(\int_{\partial \Omega \backslash \mathbb{B}_{\varepsilon R}} r^{2 \varkappa}\left(\left|\partial_{s} \partial_{n} \widetilde{u}_{0}(0, s)\right|^{2}+r^{-2}\left|\partial_{n} \widetilde{u}_{0}(0, s)\right|^{2}\right) d s \int_{0}^{\infty}\left(1+\varepsilon^{-2} n^{2}\right) e^{-2 n / \varepsilon} d n\right)^{1 / 2} \\
& \leq c \varepsilon^{3 / 2}\left(1+\varepsilon^{\varkappa-\sigma+1 / 2}\right)\left\|f ; V_{\sigma}^{1}(\Omega)\right\|\left\|\mathbf{R}_{\varepsilon} ; H^{1}(\Omega(\varepsilon))\right\| .
\end{aligned}
$$

By (41), we have the estimate

$$
\begin{aligned}
& \left|\Delta_{x} \chi^{0}(x) \widetilde{z}\left(\frac{n}{\varepsilon}, \frac{s}{\varepsilon}\right)\right| \\
& \leq c \mid\left[\Delta_{x}, \chi^{0}(x)\right] \widetilde{z}\left(\frac{n}{\varepsilon}, \frac{s}{\varepsilon}\right)+\chi(x)\left(A^{-1}(n, s) \frac{\partial A}{\partial n}(n, s) \frac{\partial \widetilde{z}}{\partial n}\left(\frac{n}{\varepsilon}, \frac{s}{\varepsilon}\right)\right. \\
& \left.\quad+\left(A(n, s)^{-2}-1\right) \frac{\partial^{2} \widetilde{z}}{\partial s^{2}}\left(\frac{n}{\varepsilon}, \frac{s}{\varepsilon}\right)-A^{-3}(n, s) \frac{\partial A}{\partial s}(n, s) \frac{\partial \widetilde{z}}{\partial s}\left(\frac{n}{\varepsilon}, \frac{s}{\varepsilon}\right)\right) \mid \\
& \leq c\left(\varepsilon^{-1}+\varepsilon^{-2} n\right) e^{-n / \varepsilon}
\end{aligned}
$$

because $\left|1-A(n, s)^{-2}\right| \leq c n$. Therefore, for the third term $I_{3}$ we have

$$
\begin{aligned}
\left|I_{3}\right| & \leq c \varepsilon\left\|\mathbf{R}_{\varepsilon} ; H^{1}(\Omega(\varepsilon))\right\|\left(\int_{\partial \Omega \backslash \mathbb{B}_{\varepsilon R}} r^{2 \varkappa}\left|\partial_{n} \widetilde{u}_{0}(0, s)\right|^{2} d s \int_{0}^{\infty} n^{2}\left(\varepsilon^{-1}+\varepsilon^{-2} n\right)^{2} e^{-2 n / \varepsilon} d n\right)^{1 / 2} \\
& \leq c \varepsilon^{3 / 2}\left(1+\varepsilon^{\varkappa-\sigma+1 / 2}\right)\left\|f ; V_{\sigma}^{1}(\Omega)\right\|\left\|\mathbf{R}_{\varepsilon} ; H^{1}(\Omega(\varepsilon))\right\| .
\end{aligned}
$$

Note that for $\varkappa=0$ the above estimates ensure the majorant (38) for the modulus of expression (39), which was used in the proof of Lemma 3.

Collecting the inequalities obtained, we arrive at the desired relation (64). 


\section{§6. MOdeling FOR $\alpha<\pi$}

Observe that, by $(6),(23)$, and $(21)$, the $L_{2}(\Omega(\varepsilon))$-norm of the last term on the right in (30) is dominated by the quantity

$$
c \varepsilon\left(\int_{0}^{d} e^{-n / \varepsilon} d n\right)^{1 / 2}\left\|\partial_{n} u_{0} ; L_{2}(\Gamma)\right\| \leq c \varepsilon^{3 / 2}\left\|f ; V_{\sigma}^{1}(\Omega)\right\| .
$$

Thus, if we restrict ourselves to approximating the solution $u_{\varepsilon}$ in $L_{2}(\Omega(\varepsilon))$-norm, we can remove the boundary layer type term from the approximate solution (30) and put it into the asymptotic remainder.

Relations (7) and (25) show that the function $u_{0}-\varepsilon m(\Pi) u^{\prime}$ leaves a small discrepancy in the third boundary-value problem

$$
-\Delta_{x} u_{\varepsilon}^{\bullet}(x)=f(x), \quad x \in \Omega, \quad \varepsilon m(\Pi) \partial_{n} u_{\varepsilon}^{\bullet}(x)+u_{\varepsilon}^{\bullet}(x)=0, \quad x \in \Gamma \backslash \mathcal{O}
$$

(cf. [18]). The boundary condition arising here is called a wall condition; such conditions for various elliptic boundary-value problems occurred in the papers [3]-[6] and elsewhere. Unfortunately, in the case under study such a simple approach does not make a success because $m(\Pi)>0$ (see Lemma 2) and so, for small $\varepsilon>0$, the left-hand side of the integral identity (see [18]) corresponding to problem (69),

$$
\left(\nabla_{x} u_{\varepsilon}^{\bullet}, \nabla_{x} v\right)_{\Omega}-\varepsilon^{-1} m(\Pi)^{-1}\left(u_{\varepsilon}^{\bullet}, v\right)_{\Gamma}=(f, v)_{\Omega}, \quad v \in H^{1}(\Omega),
$$

is not a scalar product in $H^{1}(\Omega)$ (recall that the normal $n$ is inward). Thus, there is an infinitely small sequence $\left\{\varepsilon_{n}\right\}$ of values ${ }^{4}$ of the parameter $\varepsilon$ for which problem (69) is not uniquely solvable.

Remark 4. The conclusion above is supported by the following indirect evidence. If the left-hand side in $(70)$ is a scalar product in the space $H^{1}(\Omega)$ (e.g., $m(\Pi)<0$; see Remark $5)$, then the energy functional

$$
\mathcal{E}_{\varepsilon}^{\bullet}\left(u_{\varepsilon}^{\bullet} ; \Omega\right)=\frac{1}{2} \int_{\Omega}\left|\nabla_{x} u_{\varepsilon}^{\bullet}(x)\right|^{2} d x-\frac{1}{2} \varepsilon^{-1} m(\Pi)^{-1} \int_{\Gamma}\left|u_{\varepsilon}^{\bullet}(x)\right|^{2} d s-\int_{\Omega} f(x) u_{\varepsilon}^{\bullet}(x) d x
$$

attains its minimum at the weak solution $u_{\varepsilon}^{\bullet} \in H^{1}(\Omega)$ of problem (69). The requirement $\Omega(\varepsilon) \subset \Omega$, imposed in Section 1, allows us to interpret the inclusion $\stackrel{\circ}{H}^{1}(\Omega(\varepsilon)) \subset \stackrel{\circ}{H^{1}}(\Omega)$; therefore, the energy functional

$$
\mathcal{E}_{\varepsilon}\left(u_{\varepsilon} ; \Omega(\varepsilon)\right)=\frac{1}{2} \int_{\Omega(\varepsilon)}\left|\nabla_{x} u_{\varepsilon}(x)\right|^{2} d x-\int_{\Omega(\varepsilon)} f(x) u_{\varepsilon}(x) d x
$$

for problem (2) should be minimized on a smaller set than that for the functional $\mathcal{E}\left(u_{0} ; \Omega\right)$ of problem (7), that is,

$$
\mathcal{E}\left(u_{0} ; \Omega\right) \leq \mathcal{E}_{\varepsilon}\left(u_{\varepsilon} ; \Omega(\varepsilon)\right) .
$$

Thus, if problem (69) yields a better approximation to the solution $u_{\varepsilon}$ than the limiting problem (7), then, necessarily,

$$
\mathcal{E}\left(u_{0} ; \Omega\right) \leq \mathcal{E}_{\varepsilon}^{\bullet}\left(u_{\varepsilon}^{\bullet} ; \Omega\right)=\min \left\{\mathcal{E}_{\varepsilon}^{\bullet}(v ; \Omega) \mid v \in H^{1}(\Omega)\right\} \leq \mathcal{E}_{\varepsilon}^{\bullet}\left(u_{0} ; \Omega\right) .
$$

On the other hand, we have $\mathcal{E}_{\mathcal{\varepsilon}}^{\bullet}\left(u_{0} ; \Omega\right)=\mathcal{E}\left(u_{0} ; \Omega\right)$, due to the boundary condition $u_{0}=0$ on $\Gamma$. Consequently, $u_{0}$ solves problem (69) and $\partial_{n} u_{0}=0$ on $\Gamma$. However, the maximum principle shows that this is impossible if $f>0$. This contradiction means that in the case where $\Omega(\varepsilon) \subset \Omega$, the left-hand side of identity (70) cannot be a scalar product.

\footnotetext{
${ }^{4}$ The quantities $\varepsilon_{n}^{-1} m(\Pi)^{-1}>0$ are the positive eigenvalues of the Steklov spectral problem.
} 


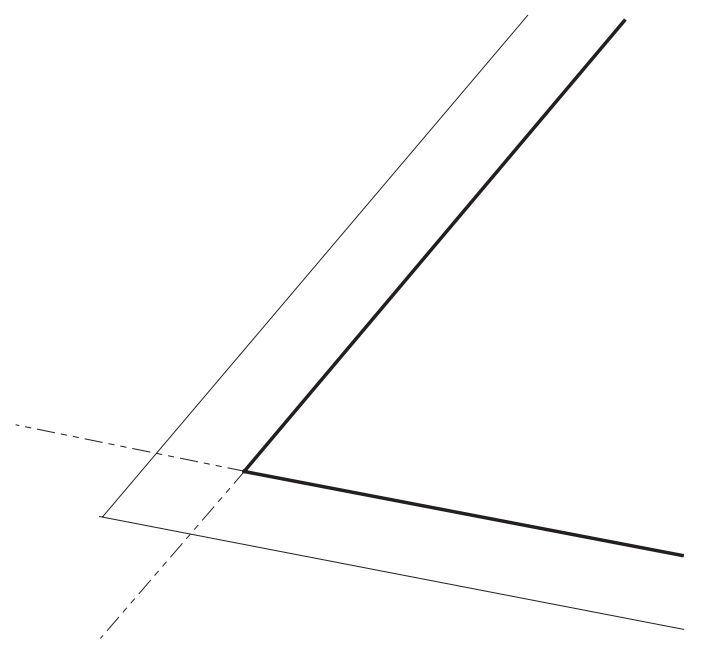

FiguRE 6

Remark 5. Consider the half-strip $\Pi^{t}=\left\{\eta:\left(\eta_{1}+t, \eta_{2}\right) \in \Pi\right\}$, depending on a parameter $t$. It is easily seen that the special solution $z^{t}$ (indicated in Lemma 1) of the homogeneous problem (4) in $\Pi^{t}$ has the form $z^{t}(\eta)=z\left(\eta_{1}+t, \eta_{2}\right)$, i.e., by (6),

$$
z^{t}(\eta)=\eta_{1}+t-m(\Pi)+O\left(e^{-\eta_{1}}\right)
$$

which implies that the quantity $m\left(\Pi^{t}\right)=m(\Pi)-t$ is negative for large $t$. So, if the inclusion $\Omega(\varepsilon) \subset \Omega$ fails, then the left-hand side of (70) can be a scalar product in $H^{1}(\Omega)$, and the third boundary-value problem (69) can be uniquely solvable. In this case the application of wall boundary conditions is meaningful.

The concept of a smooth image of the perturbed boundary (see $[9,3,10]$ ) allows us to preserve the differential equation and the type of the boundary condition when replacing the periodic curve $\Gamma(\varepsilon)$ with a curve $\Gamma_{\varepsilon}^{\#}$ differing little from the contour $\Gamma=\partial \Omega$. Namely, we define an $\operatorname{arc} \Gamma_{\varepsilon}^{\#}$ off the disk $\mathbb{B}_{R^{0} / 2}$ by the formula

$$
\Gamma_{\varepsilon}^{\#}=\left\{x \in \mathcal{V}_{d} \backslash \mathbb{B}_{R^{0} / 2}: n=m(\Pi) \varepsilon\right\},
$$

and inside $\mathbb{B}_{R^{0}}$ we let $\Gamma_{\varepsilon}^{\#}$ coincide with the boundary of the angle

$$
\mathbb{K}_{\varepsilon}^{\#}=\bigcap_{ \pm}\left\{x: r \sin \left(\frac{\alpha}{2} \mp \varphi\right)>m(\Pi) \varepsilon\right\}
$$

Note that the angle (73) is obtained from $\mathbb{K}$ using parallel translation of the sides $\{x: r>0, \varphi= \pm \alpha / 2\}$ by the distance of $m(\Pi) \varepsilon$ (see Figure 6 ). Since $m(\Pi)>0$, the domain $\Omega_{\varepsilon}^{\#}$ enclosed by the contour $\Gamma_{\varepsilon}^{\#}$ lies strictly inside $\Omega$. The vertex of the angle $\mathbb{K}_{\varepsilon}^{\#}$ will be denoted by $\mathcal{O}_{\varepsilon}^{\#}$.

In the domain $\Omega_{\varepsilon}^{\#}$, we consider the Dirichlet problem

$$
-\Delta_{x} u_{\varepsilon}^{\#}(x)=f(x), \quad x \in \Omega_{\varepsilon}^{\#}, \quad u_{\varepsilon}^{\#}(x)=0, x \in \Gamma_{\varepsilon}^{\#} \backslash \mathcal{O}_{\varepsilon}^{\#} .
$$

It can be assumed that $\Omega_{\varepsilon}^{\#}$ is obtained from the limiting domain $\Omega$ by the rule described in Section 1: it suffices to take the constant $m(\Pi)$ for the role of the function $H$ occurring in (1). Therefore, all the results obtained for problem (2) remain valid also for problem (74). Moreover, the solution $z^{\#}$ of the homogeneous problem (4) in the halfstrip $\Pi^{\#}=\left\{\eta \in \mathbb{R}^{2}: \eta_{1}>m(\Pi), \eta_{2} \in(0,2 \pi)\right\}$ corresponding to the boundary (72) is of the form $z^{\#}(\eta)=\eta_{1}-m(\Pi)$, i.e., we have $m\left(\Pi^{\#}\right)=m(\Pi)$. Hence, the global asymptotic 
approximations $U_{\varepsilon}$ and $U_{\varepsilon}^{\#}$ to the solutions $u_{\varepsilon}$ and $u_{\varepsilon}^{\#}$ of problems (2) and (74) differ from each other only in a boundary layer ${ }^{5}$ the norm of which in the space $L_{2}(\Omega(\varepsilon))$ is dominated by the quantity (68). It remains to formulate the result.

Proposition 1. Suppose $\alpha<\pi$ and conditions (21) are fulfilled. Then the solutions $u_{\varepsilon}$ and $u_{\varepsilon}^{\#}$ of the Dirichlet problems (2) and (73), extended by zero to $\Omega$, satisfy the inequality

$$
\left\|u_{\varepsilon}-u_{\varepsilon}^{\#} ; L_{2}(\Omega)\right\| \leq c \varepsilon^{\min \{2-\sigma, 3 / 2\}}\left\|f ; V_{\sigma}^{1}(\Omega)\right\|,
$$

where $c$ is a constant independent of $\varepsilon \in\left(0, \varepsilon_{0}\right]$ and of the right-hand side $f$ of the two problems.

\section{$\S 7$. Modeling for $\alpha \in(\pi, 2 \pi)$ : Selfadjoint extensions}

We should at once give up using wall boundary conditions, because the harmonic function $\zeta$ occurring in the asymptotic construction (56) fails to satisfy problem (69) due to bad singularity (10) at the corner point: the solution of the third boundary-value problem, as well as of the Neumann problem, must start with the term $\pi \chi(r) r^{-\pi / \alpha} \sin (\pi \varphi / \alpha)$ (see the introductory Chapter 2 in [27]).

For the modeling of problem (2), a key observation is the following: the $L_{2}(\Omega(\varepsilon)$ )norm of the power-law boundary layer (the next to the last term on the right in (51)) is sufficiently small because, in accordance with Remark 2 and estimate (51), we have

$$
\varepsilon^{\pi / \alpha}|K|\left(\int_{\Omega(\varepsilon)} \chi(r)^{2}\left|\widetilde{Y}\left(\varepsilon^{-1} x\right)\right|^{2} d x\right)^{1 / 2} \leq c \varepsilon^{1+\pi / \alpha}\left(1+\delta_{\alpha, 2 \pi}|\log \varepsilon|\right)^{1 / 2}\left\|f ; V_{\sigma}^{1}(\Omega)\right\| .
$$

Corollary 1. Let $f \in V_{1}^{1}(\Omega) \subset L_{2}(\Omega)$. Then, for any arbitrarily small $\delta>0$, we have

$$
\left\|u_{\varepsilon}-\left(u_{0}-\varepsilon m(\Pi) u_{0}^{\prime}+a_{0}(\varepsilon) \zeta\right) ; L_{2}(\Omega(\varepsilon))\right\| \leq c_{\delta} \varepsilon^{\min \{1-\delta+\pi / \alpha, 3 / 2\}}\left\|f ; V_{1}^{1}(\Omega)\right\|,
$$

where the constant $c_{\delta}$ depends on the chosen number $\delta>0$ but is independent of the parameter $\varepsilon \in\left(0, \varepsilon_{0}\right]$ and of the function $f$.

Proof. We fix the weight indices $\varkappa=\alpha^{-1} \pi-\delta / 2$ and $\sigma=\max \left\{1,2\left(1-\alpha^{-1} \pi\right)+\delta / 2\right\}$; then $V_{1}^{1}(\Omega) \subset V_{\sigma}^{1}(\Omega)$ and $\left\|u_{\varepsilon}-U_{\varepsilon} ; L_{2}(\Omega(\varepsilon))\right\| \leq c\left\|u_{\varepsilon}-U_{\varepsilon} ; V_{\varkappa}^{1}(\Omega(\varepsilon))\right\|$, because $\varkappa-1<0$. Now estimate (75) follows from Theorem 3.

If $\alpha \in(\pi, 2 \pi)$, then we can find $\delta>0$ for which the exponent of $\varepsilon$ on the right in (75) is equal to $3 / 2$. Now we analyze precisely this case, postponing the consideration of the domain with a cut $(\alpha=2 \pi)$ till the next section.

As in Section 6 , we define the boundary of $\Gamma_{\varepsilon}^{\#}$ off the disk $\mathbb{B}_{\mathbb{R}^{0} / 2}$ by formula (72) and assume that inside the disk $\mathbb{B}_{R^{0}}$ the boundary of $\Omega_{\varepsilon}^{\#}$ coincides with the union of the sides of the angle

$$
\mathbb{K}_{\varepsilon}^{\#}=\bigcup_{ \pm}\left\{x: r \sin \left(\frac{\alpha}{2} \mp \varphi\right)>m(\Pi) \varepsilon\right\}
$$

(see Figure 7 and compare with (73)). To describe the asymptotics of the solution of problem (74) in the angular domain $\Omega_{\varepsilon}^{\#}$, we need to study the solution (15) of the homogeneous problem (11) in the angle $\Xi^{\#}=\left\{\xi \in \mathbb{R}^{2}: \varepsilon \xi \in \mathbb{K}_{\varepsilon}^{\#}\right\}$, which is obtained from $\mathbb{K}$ by shifting its vertex by the distance of $d_{\alpha}(\Pi)=(\sin (\alpha / 2))^{-1} m(\Pi)$ along the bisector of $\mathbb{K}$. It is clear that

$$
Y(\xi)=\rho_{1}^{\pi / \alpha} \cos \left(\pi \varphi_{1} / \alpha\right)
$$

\footnotetext{
${ }^{5}$ In the asymptotics of the solution of problem (74), no exponential boundary layer is present, because the perturbation of the boundary is regular far from the corner point (see $[29, \S 7.6 .5$, p. 528]).
} 




FIGURE 7

here $\left(\rho_{1}, \varphi_{1}\right)$ is the polar coordinate system centered at $\left(d_{\alpha}(\Pi), 0\right)$ and with polar axis directed along the bisector of $\mathbb{K}$. At the same time, by the general results of the theory of boundary-value problems in domains with piecewise smooth boundary, we have the following expansion, which is simpler than (16) because no boundary layer is present:

$$
\begin{aligned}
Y(\xi)= & \varrho^{\pi / \alpha} \cos \left(\frac{\pi}{\alpha} \varphi\right)+\frac{\pi}{\alpha} m(\Pi) Y_{1}(\xi)-m\left(\Xi^{\#}\right) \rho^{-\pi / \alpha} \cos \left(\frac{\pi}{\alpha} \varphi\right) \\
& +O\left(\rho^{-\max \{2 \pi / \alpha, 2-\pi / \alpha\}}\right), \quad \rho \rightarrow \infty .
\end{aligned}
$$

We put $\varphi=\varphi_{1}=0$ in (77) and (78); then $\rho=\rho_{1}+d_{\alpha}(\Pi)$, whence

$$
Y(\xi, 0)=\left(\rho-d_{\alpha}(\Pi)\right)^{\pi / \alpha}=\rho^{\pi / \alpha}\left(1-\frac{\pi}{\alpha} d_{\alpha}(\Pi) \rho^{-1}+O\left(\rho^{-2}\right)\right) .
$$

Since $Y_{1}\left(\xi_{1}, 0\right)=\rho^{-1+\pi / \alpha}[\sin (\alpha / 2)]^{-1}$ by the definition (17), comparison of (79) and (78) shows that

$$
m\left(\Xi^{\#}\right)=0 .
$$

So, unlike the solution $u_{\varepsilon}$ of the initial problem (2), for the asymptotics of the solution $u_{\varepsilon}^{\#}$ of problem (74) we can take the sum $u_{0}-\varepsilon m(\Pi) u_{0}^{\prime}$, without the singular component $a_{0}(\varepsilon) \zeta$. If $\alpha \in(\pi, 4 \pi / 3]$, then the approximation accuracy $O\left(\varepsilon^{3 / 2}\right)$ allows us to exclude the weight function $\zeta$ from the asymptotic approximation indicated on the left-hand side of $(75)$, because $a_{0}(\varepsilon)=O\left(\varepsilon^{2 \pi / \alpha}\right)$ decays at the same rate or more rapidly (see $\left.(48)\right)$. As a result, applying Corollary 1 to both problems (2) and (74), we arrive at the next statement.

Proposition 2. Suppose $\alpha \in\left(\pi, \frac{4}{3} \pi\right]$ and $f \in V_{1}^{1}(\Omega)$. Then the solutions $u_{\varepsilon} \in \stackrel{\circ}{H^{1}}(\Omega(\varepsilon))$ and $u_{\varepsilon}^{\#} \in \stackrel{\circ}{H^{1}}\left(\Omega_{\varepsilon}^{\#}\right)$ of problems (2) and (74), extended by zero to $\Omega$, satisfy the inequality

$$
\left\|u_{\varepsilon}-u_{\varepsilon}^{\#} ; L_{2}(\Omega)\right\| \leq c \varepsilon^{3 / 2}\left\|f ; V_{1}^{1}(\Omega)\right\|,
$$

with a constant $c$ independent of the parameter $\varepsilon \in\left(0, \varepsilon_{0}\right]$ and of the function $f$. 
In the case where $\alpha \in(4 \pi / 3,2 \pi)$, the absence of the singular component reduces the asymptotic accuracy of modeling. In order to include the weight function in the solution of problem (74), we apply the techniques of selfadjoint extensions. Namely, in the space $L_{2}\left(\Omega_{\varepsilon}^{\#}\right)$ we consider the unbounded operator $A_{0}$ given by the differential expression $-\Delta_{x}$ and having the domain

$$
\mathcal{D}\left(A_{0}\right)=\left\{v \in C_{0}^{\infty}\left(\overline{\Omega_{\varepsilon}^{\#}} \backslash O_{\varepsilon}^{\#}\right): v=0 \text { on } \Gamma_{\varepsilon}^{\#} \backslash O_{\varepsilon}^{\#}\right\} .
$$

The following fairly simple fact is well known (see, e.g., [30, 21]): the domains of the closure $A$ for the operator $A_{0}$ and of the adjoint operator $A^{*}$ look like this:

$$
\begin{aligned}
\mathcal{D}(A) & =\left\{v \in V_{0}^{\#}\left(\Omega_{\varepsilon}^{\#}\right): v=0 \text { on } \Gamma_{\varepsilon}^{\#} \backslash O_{\varepsilon}^{\#}\right\}, \\
& =\mathcal{D}\left(A^{*}\right)=\left\{v(x)=v_{0}^{(x)}+a^{\#} \zeta^{\#}(x)+\chi\left(r_{\varepsilon}\right) K^{\#} r_{\varepsilon}^{\pi / \alpha} \cos \pi \varphi_{\varepsilon} / \alpha:\right. \\
& \left.\quad v_{0} \in \mathcal{D}(A), a^{\#}, K^{\#} \in \mathbb{R}\right\} .
\end{aligned}
$$

Here $\zeta^{\#} \in L_{2}\left(\Omega_{\varepsilon}^{\#}\right)$ is the weight function for the domain $\Omega_{\varepsilon}^{\#}$, i.e., the solution of the homogeneous problem (74), admitting representation in the form

$$
\zeta^{\#}(x)=\frac{1}{\pi} \chi\left(r_{\varepsilon}\right) r_{\varepsilon}^{-\pi / \alpha} \cos \left(\frac{\pi}{\alpha} \varphi_{\varepsilon}\right)+\zeta_{0}^{\#}(x), \quad \zeta_{0}^{\#} \in \stackrel{\circ}{H}^{1}\left(\Omega_{\varepsilon}^{\#}\right),
$$

where $\left(r_{\varepsilon}, \varphi_{\varepsilon}\right)$ is the polar coordinate system centered at $\mathcal{O}_{\varepsilon}^{\#}$ and with polar axis directed along the bisector of the angle (76), and the weighted norm in the space $V_{\sigma}^{\#}\left(\Omega_{\varepsilon}^{\#}\right)$ has the form (20) with the replacement of the factor $r$ by the factor $r_{\varepsilon}=\left|x-\mathcal{O}_{\varepsilon}^{\#}\right|$. The next statement is also well known (see [31] and also [30, 21]).

Lemma 7. Let $\vartheta \in[0, \pi)$. The restriction $\mathcal{A}(\vartheta)$ of the operator $A^{*}$ to the linear space

$$
\mathcal{D}(\mathcal{A}(\vartheta))=\left\{v \in \mathcal{D}\left(A^{*}\right): a^{\#} \cos \vartheta+K^{\#} \sin \vartheta=0\right\}
$$

is a selfadjoint extension of $A$. Every selfadjoint extension of $A$ has a domain of the form (80) with some $\vartheta \in[0, \pi)$.

Remark 6 . The proof of Lemma 7 can be based on the following observation: for any $u, v \in \mathcal{D}(\mathcal{A}(\vartheta))$ we have

$$
\begin{aligned}
\left(A^{*} v\right. & , u)_{\Omega_{\varepsilon}^{\#}}-\left(v, A^{*} u\right)_{\Omega_{\varepsilon}^{\#}} \\
& =\left(-\Delta_{x} v^{\#}, u^{\#}+a_{u}^{\#} \zeta^{\#}\right)_{\Omega_{\varepsilon}^{\#}}-\left(v^{\#}+a_{v}^{\#} \zeta^{\#},-\Delta_{x} u^{\#}\right)_{\Omega_{\varepsilon}^{\#}} \\
& =\left(v^{\#}, \Delta_{x} u^{\#}\right)_{\Omega_{\varepsilon}^{\#}}-\left(\Delta_{x} v^{\#}, u^{\#}\right)_{\Omega_{\varepsilon}^{\#}}+a_{v}^{\#}\left(\zeta^{\#}, \Delta_{x} u^{\#}\right)_{\Omega_{\varepsilon}^{\#}}-a_{u}^{\#}\left(\Delta_{x} v^{\#}, \zeta^{\#}\right)_{\Omega_{\varepsilon}^{\#}} \\
& =a_{u}^{\#} K_{v}^{\#}-a_{v}^{\#} K_{u}^{\#}=0 .
\end{aligned}
$$

Here

$$
v^{\#}=v_{0}+\chi K_{v}^{\#} r_{\varepsilon}^{\pi / \alpha} \cos \left(\pi \varphi_{\varepsilon} / \alpha\right) \in \stackrel{\circ}{H^{1}}\left(\Omega_{\varepsilon}^{\#}\right),
$$

and the function $u^{\#} \in \stackrel{\circ}{H}^{1}\left(\Omega_{\varepsilon}^{\#}\right)$ is defined similarly; the Green formula was applied precisely to this pair of functions in the calculation (81). The last identity in (81) is valid due to the constraint imposed in (80) on the pairs of coefficients $a_{v}^{\#}, K_{v}^{\#}=-\left(\Delta_{x} v^{\#}, \zeta^{\#}\right)_{\Omega_{\varepsilon}^{\#}}$ and $a_{u}^{\#}, K_{u}^{\#}=-\left(\Delta_{x} u^{\#}, \zeta^{\#}\right)_{\Omega_{\varepsilon}^{\#}}$. Also, we have used the integral representation (9) in the domain $\Omega_{\varepsilon}^{\#}$.

Recalling formula (48), we introduce the selfadjoint extension parameter

$$
\vartheta_{\alpha}(\Xi)=\arctan \left(\varepsilon^{2 \pi / \alpha} \pi m(\Xi)\right) \text {. }
$$

Then the solution of the abstract equation

$$
\mathcal{A}\left(\vartheta_{\alpha}(\Xi)\right) v=f \in L_{2}\left(\Omega_{\varepsilon}^{\#}\right)
$$


takes the form

$$
v(x)=v^{\#}(x)-\varepsilon^{2 \pi / \alpha} \pi K^{\#} m(\Xi) \zeta^{\#}(x),
$$

where $v^{\#} \in \stackrel{\circ}{H}^{1}\left(\Omega_{\varepsilon}^{\#}\right)$ is the solution of problem (74), and $K^{\#}$ is the coefficient in the expansion

$$
v^{\#}(x)=K^{\#} \chi\left(r_{\varepsilon}\right) r_{\varepsilon}^{\pi / \alpha} \cos \left(\frac{\pi}{\alpha} \varphi_{\varepsilon}\right)+\widetilde{v}^{\#}(x), \quad \widetilde{v}^{\#} \in V_{0}^{2}\left(\Omega_{\varepsilon}^{\#}\right) \subset H^{2}\left(\Omega_{\varepsilon}^{\#}\right) .
$$

So, to check that the model (82) is consistent, it remains to compare the weight functions $\zeta$ and $\zeta^{\#}$.

Lemma 8. We have

$$
\left\|\zeta-\zeta^{\#} ; L_{2}(\Omega)\right\| \leq c \varepsilon^{1-\pi / \alpha}
$$

Proof. We can write

$$
\left\|\zeta ; L_{2}\left(\Omega \cap \mathbb{B}_{\varepsilon R}\right)\right\|^{2}+\left\|\zeta^{\#} ; L_{2}\left(\Omega_{\varepsilon}^{\#} \cap \mathbb{B}_{\varepsilon R}\right)\right\|^{2} \leq c \int_{0}^{\varepsilon\left(R+d_{\alpha}(\Pi)\right)} r^{-2 \pi / \alpha} r d r \leq c \varepsilon^{2(1-\pi / \alpha)} .
$$

Since $\zeta \in C^{\infty}(\bar{\Omega} \backslash \mathcal{O})$ and the function $x \mapsto \zeta^{\varepsilon}(x)=\zeta\left(x_{1}-\varepsilon d_{\alpha}(\Pi), x_{2}\right)$, extended smoothly to the domain $\Omega_{\varepsilon}^{\#}$, is harmonic on the set $\left\{x:\left(x_{1}+\varepsilon d_{\alpha}(\Pi), x_{2}\right) \in \Omega\right\}$ and vanishes on the boundary $\partial \Omega_{\varepsilon}^{\#}$ near the corner point, the difference $\zeta^{\varepsilon}-\zeta^{\#}$ is an energy solution (the singularities cancel) of the Dirichlet problem in $\Omega_{\varepsilon}^{\#}$ with data $f_{\varepsilon}^{\#}$ and $g_{\varepsilon}^{\#}$, where $f_{\varepsilon}^{\#}=0$ and $g_{\varepsilon}^{\#}=0$ near the vertex $\mathcal{O}_{\varepsilon}^{\#}$ and

$$
\left\|f_{\varepsilon}^{\#} ; L_{2}\left(\Omega_{\varepsilon}^{\#}\right)\right\|+\left\|g_{\varepsilon}^{\#} ; H^{1 / 2}\left(\Omega_{\varepsilon}^{\#}\right)\right\| \leq c \varepsilon .
$$

Thus, we have

$$
\left\|\zeta^{\varepsilon}-\zeta^{\#} ; H^{1}\left(\Omega_{\varepsilon}^{\#}\right)\right\| \leq c \varepsilon
$$

Consequently,

$$
\begin{aligned}
\left\|\zeta-\zeta^{\#} ; L_{2}\left(\Omega \backslash \mathbb{B}_{\varepsilon R}\right)\right\|^{2} & \leq c\left(\varepsilon^{2}+\left\|\zeta-\zeta^{\varepsilon} ; L_{2}\left(\Omega \backslash \mathbb{B}_{\varepsilon R}\right)\right\|^{2}\right) \\
& \leq c \varepsilon^{2}\left(1+\int_{\Omega \backslash \mathbb{B}_{\varepsilon R}}\left|\nabla_{x} \zeta(x)\right|^{2} d x\right) \\
& \leq c \varepsilon^{2}\left(1+\int_{\varepsilon R}^{\operatorname{diam} \Omega} r^{-2-2 \pi / \alpha} r d r\right) \leq c \varepsilon^{2(1-\pi / \alpha)} .
\end{aligned}
$$

We now present the main result of this section.

Proposition 3. Suppose $\alpha \in\left(\frac{4}{3} \pi, 2 \pi\right)$ and $f \in V_{1}^{1}(\Omega)$. Then the solution $u_{\varepsilon} \in \stackrel{\circ}{H}^{1}(\Omega(\varepsilon))$ of problem (2) and the solution $v_{\varepsilon} \in L_{2}\left(\Omega_{\varepsilon}^{\#}\right)$ of the abstract equation (82), both extended by zero to the domain $\Omega$, satisfy the inequality

$$
\left\|u_{\varepsilon}-v_{\varepsilon} ; L_{2}(\Omega)\right\| \leq c_{\alpha} \varepsilon^{3 / 2}\left\|f ; V_{1}^{1}(\Omega)\right\|
$$

with a constant $c$ depending on $\alpha$, but independent of $\varepsilon \in\left(0, \varepsilon_{0}\right]$ and of $f \in V_{1}^{1}(\Omega) \subset$ $L_{2}(\Omega)$.

Proof. When referring to Theorem 3, we assume that the weight indices $\sigma$ and $\varkappa$ are chosen as was indicated in the proof of Corollary 1 and in the comments after it. By that corollary applied to problems (2) and (74), we have the approximate identities

$$
\begin{aligned}
& u_{\varepsilon} \approx u_{0}-\varepsilon m(\Pi) u_{0}^{\prime}-\varepsilon^{2 \pi / \alpha} \pi K m(\Xi) \zeta, \\
& v_{\varepsilon}^{\#}=u_{\varepsilon}^{\#} \approx u_{0}-\varepsilon m(\Pi) u_{0}^{\prime}
\end{aligned}
$$




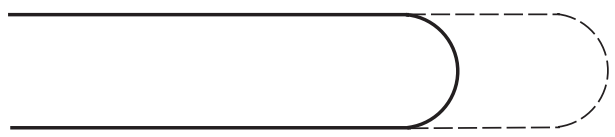

Figure 8

and in both cases the accuracy of approximation is equal to the majorant in (85). By Lemma 8 and the integral representation (9) of the intensity coefficients, we have

$$
\begin{aligned}
\left|K-K^{\#}\right| & =\left|\int_{\Omega} f(x) \zeta(x) d x-\int_{\Omega_{\varepsilon}^{\#}} f(x) \zeta^{\#}(x) d x\right| \\
& \leq \int_{\Omega_{\varepsilon}^{\#}}|f(x)|\left|\zeta(x)-\zeta^{\#}(x)\right| d x+\int_{\Omega \backslash \Omega_{\varepsilon}^{\#}}|f(x)| \zeta(x) d x \\
\leq & c\left\|f ; L_{2}(\Omega)\right\|\left(\left\|\zeta-\zeta^{\#} ; L_{2}(\Omega)\right\|^{2}\right. \\
& \left.\quad+\int_{\Omega \cap \mathbb{B}_{\varepsilon R}} r^{-2 \pi / \alpha} d x+\int_{\Omega \backslash\left(\Omega_{\varepsilon}^{\#} \cup \mathbb{B}_{\varepsilon R}\right)} r^{-2 \pi / \alpha} \varrho(x)^{2} d x\right)^{1 / 2} \\
& \leq c \varepsilon^{1-\pi / \alpha}\left\|f ; L_{2}(\Omega)\right\| .
\end{aligned}
$$

Explanation: when treating the integral over the set $\Omega \backslash \Omega_{\varepsilon}^{\#}$, we applied the obvious relations

$$
0 \leq \zeta(x) \leq c r^{-\pi / \alpha} \quad \text { and } \quad \zeta(x) \leq C r^{-\pi / \alpha} \varrho(x)
$$

for the weight function (10); here $\varrho(x)$ is the quantity (29).

Now, the required estimate of the $L_{2}(\Omega)$-norm for the difference of the expressions $\varepsilon^{2 \pi / \alpha} \pi K m(\Xi) \zeta$ and $\varepsilon^{2 \pi / \alpha} \pi K^{\#} m(\Xi) \zeta^{\#}$ (see (83) and (86)) follows from inequalities (84) and (87).

\section{$\S 8$. Modeling for $\alpha=2 \pi$ : Singular perturbation of the boundary}

For clear reasons, in the case of a cut, the sets (73) and (76) are not angles of opening $2 \pi$. Therefore, for the role of the angle domain $\Xi$ that describes the boundary of $\Omega_{\varepsilon}^{\#}$ near the point $\mathcal{O}$ we take the set $\Xi^{t}=\mathbb{R}^{2} \backslash \overline{G^{t}}$; this is a half-plane from which a half-strip with curvilinear end is removed. Here $G^{t}=\left\{\xi:\left(\xi_{1}-t, \xi_{2}\right) \in G^{0}\right\}$, and $G^{0}$ is a set that coincides with the half-strip $\left\{\xi: \xi_{1}<0,\left|\xi_{2}\right|<m(\Pi)\right\}$ off the ball $\mathbb{B}_{R}$ and has a smooth (for simplicity) boundary $\partial G^{0}$ (see Figure 8, where the boundaries $\partial G^{0}$ and $\partial G^{t}$ are depicted by the solid line and the dashed line, respectively).

Lemma 9. For the solution $Y^{t}$ of the homogeneous problem (11), given by formula (15) with $\alpha=2 \pi$, we have the expansion

$$
Y^{t}(\xi)=\rho^{1 / 2} \cos \left(\frac{\varphi}{2}\right)-\frac{1}{2} m(\Pi) Y_{1}(\xi)-m\left(\Xi^{t}\right) \rho^{-1 / 2} \cos \left(\frac{\varphi}{2}\right)+O\left(\rho^{-1}\right), \quad \rho \rightarrow \infty,
$$

where $Y_{1}$ is the function (17). Moreover,

$$
m\left(\Xi^{t}\right)=m\left(\Xi^{0}\right)-t / 2 .
$$

Proof. Since in the neighborhood of infinity the domain $\Xi^{t}$ differs little from an angle of opening $2 \pi$ (i.e., the plane with a cut), formula (88) is a consequence of the general results of the theory of boundary-value problems in conic (angular) domains (see [24] 


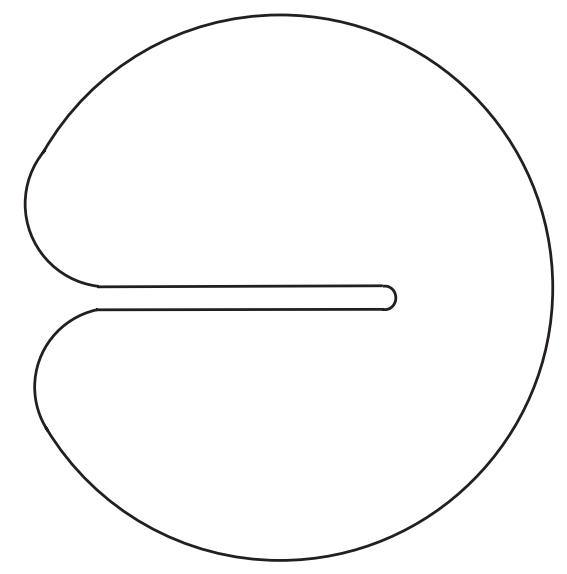

Figure 9

and, e.g., [27, Chapter 2]). By the definition of $\Xi^{t}$, we have $Y^{t}(\xi)=Y^{0}\left(\xi_{1}+t, \xi_{2}\right)$. Putting $\varphi=0, \xi_{2}=0$, and using (17), we obtain

$$
\begin{aligned}
Y^{t}(\rho, 0) & =Y^{0}(\rho+t, 0) \\
& =(\rho+t)^{1 / 2}-(2 \pi)^{-1} m(\Pi)(\rho+t)^{1 / 2} \log (\rho+t)-m\left(\Xi^{0}\right)(\rho+t)^{1 / 2}+O\left((\rho+t)^{-1}\right) \\
& =\rho^{1 / 2}+\frac{t}{2} \rho^{-1 / 2}-\frac{1}{2 \pi} m(\Pi) \rho^{-1 / 2} \log \rho-m\left(\Xi^{0}\right) \rho^{-1 / 2}+O\left(\rho^{-1}\right), \quad \rho \rightarrow \infty .
\end{aligned}
$$

Comparing this with relation (88), where $\xi_{2}=0$, we get the desired formula (89).

Let $\Omega_{\varepsilon, t}^{\#}$ be a domain such that off the disk $\mathbb{B}_{\varepsilon R / 2}$ the boundary $\Gamma_{\varepsilon, t}^{\#}$ of $\Omega_{\varepsilon, t}^{\#}$ is defined in the same way as in the preceding two sections, and inside the disk $\mathbb{B}_{\varepsilon R}$ the domain $\Omega_{\varepsilon, t}^{\#}$ coincides with the set $\left\{x: \varepsilon^{-1} x \in \Xi^{t}\right\}$ (see Figure 9 ). In this case, the calculations of Section 5 (see, in particular, formula (49), and also Theorem 3 and Corollary 1), applied to problem (74) in the domain $\Omega_{\varepsilon, t}^{\#}$, show that

$$
\left\|u_{\varepsilon, t}^{\#}-\left(u_{0}-\varepsilon m(\Pi) u_{0}^{\prime}-a_{0, t}^{\#}(\varepsilon) \zeta\right) ; L_{2}\left(\Omega(\varepsilon) \cap \Omega_{\varepsilon, t}^{\#}\right)\right\| \leq c_{\delta} \varepsilon^{-\delta+3 / 2}\left\|f ; V_{1}^{1}(\Omega)\right\|,
$$

where

$$
a_{0, t}^{\#}(\varepsilon)=-\varepsilon K\left(\pi m\left(\Xi^{t}\right)+\frac{1}{2} m(\Pi) \log \varepsilon\right) .
$$

Comparing the definitions (90) and (49), and using (89), we see that for

$$
t=t^{0}:=2 m\left(\Xi^{0}\right)-2 m(\Xi)
$$

the solutions of the problems in question possess one and same asymptotics. Thus, the solution of problem (74) in the domain $\Omega_{\varepsilon, t^{0}}^{\#}$ with $t$ chosen as in (91) yields the best approximation to the solution of the initial problem (2) in a domain with rapidly oscillating boundary.

Now, in general, the inclusion $\Omega_{\varepsilon, t^{0}}^{\#} \subset \Omega$ fails. Therefore, in the proposition below, which is a consequence of the preceding results, it is assumed that the right-hand side $f$ of problems (2) and (7) belongs to the space $V_{1}^{1}(\bar{\Omega})$, i.e., has no jumps on the cut.

Proposition 4. Suppose $\alpha=2 \pi, f \in V_{1}^{1}(\bar{\Omega})$, and $u_{\varepsilon, t^{0}}^{\#}$ is the solution of problem (74) in the domain $\Omega_{\varepsilon, t}^{\#}$ constructed as described above. The solution $u_{\varepsilon}$ of problem (2) satisfies 
the inequality

$$
\left\|u_{\varepsilon}-u_{\varepsilon, t^{0}}^{\#} ; L_{2}\left(\Omega(\varepsilon) \cap \Omega_{\varepsilon, t}^{\#}\right)\right\| \leq c_{\delta} \varepsilon^{-\delta+3 / 2}\left\|f ; V_{1}^{1}(\bar{\Omega})\right\|,
$$

where $c_{\delta}$ is a constant depending on an arbitrarily chosen number $\delta>0$, but independent of the parameter $\varepsilon \in\left(0, \varepsilon_{0}\right]$ and of the function $f$.

Remark 7. Modeling of problem (2) with the help of a singular perturbation of the domain near the corner point can be performed also for $\alpha \in(4 \pi / 3,2 \pi)$, i.e., in the case treated in Section 7, and then there is no need in invoking the theory of selfadjoint extensions. To this end, we take the set $\left\{\xi: t \xi \in \Xi^{0}\right\}$ for the role of the domain $\Xi^{t}$ describing the geometry of $\Gamma_{\varepsilon, t}^{\#}$ near $\mathcal{O}$; here $\Xi^{0}$ is a domain with an angular exit to infinity, i.e., outside of the disk $\mathbb{B}_{R}$ the domain $\Xi^{0}$ coincides with the angle $\mathbb{K}_{1}=\left\{\xi: \rho_{1}>0,\left|\varphi_{1}\right|<\alpha / 2\right\}$. It is not hard to check that in the expansion

$$
Y^{t}(\xi)=\rho_{1}^{\pi / \alpha} \cos \left(\pi \varphi_{1} / \alpha\right)-m\left(\Xi^{t}\right) \rho_{1}^{-\pi / \alpha} \cos (\pi \varphi / \alpha)+O\left(\rho_{1}^{-\pi / \alpha}\right), \quad \rho_{1} \rightarrow+\infty
$$

of the solution of the homogeneous problem (11) (this expansion is similar to (15), (16), but simpler), we have $m\left(\Xi^{t}\right)=t^{-2 \pi / \alpha} m\left(\Xi^{0}\right)$. Thus, by choosing the extension coefficient $t>0$ appropriately, we can ensure the required coefficient of the weight function $\zeta^{\#}$ in the asymptotics of the solution of $(74)$ in $\Omega_{\varepsilon, t}^{\#}$. The fact that $m\left(\Xi^{0}\right)$ can take both positive and negative values is verified in the next lemma; this is necessary, because the sign of the quantity $m(\Xi)$ occurring in (48) is unknown (see Remark 3).

Lemma 10. Suppose $\Xi^{0} \neq \mathbb{K}_{1}$.

1) If $\Xi^{0} \subset \mathbb{K}_{1}$, then $m\left(\Xi^{0}\right)>0$.

2) If $\mathbb{K}_{1} \subset \Xi^{0} \subset\left\{\xi: \rho_{1} \geq 0,\left|\varphi_{1}\right|<\min \{\pi, 3 \alpha / \pi\}\right\}$, then $m\left(\Xi^{0}\right)<0$.

Proof. We seek the solution (92) in the form

$$
Y^{0}(\xi)=\rho_{1}^{\pi / \alpha} \cos \left(\pi \varphi_{1} / \alpha\right)+\mathcal{Y}^{0}(\xi) .
$$

Clearly, $\mathcal{Y}^{0}$ is a harmonic function that vanishes on $\partial \Xi^{0} \backslash \mathbb{B}_{R}$ and is nonnegative in case 1) and nonpositive in case 2). The maximum principle determines the sign of that function, which can be expanded, for $\rho_{1}>R$, in a convergent series in the harmonics $\rho_{1}^{-\pi k / \alpha} \sin \left(\pi k\left(\alpha^{-1} \varphi_{1}+1 / 2\right)\right)$. The first of them $(k=1)$ is positive in the sector $\left\{\xi \in \mathbb{K}_{1}: \rho_{1}>R\right\}$, and the other harmonics $(k=2,3, \ldots)$ have variable sign. Thus, the coefficient $m\left(\Xi^{0}\right)$ of the first harmonic inherits its sign from the function $\mathcal{Y}^{0}$.

\section{§9. A FEW WORDS ABOUT APPLICATIONS}

Modeling of the boundary-value problem (2) in a domain with a singularly perturbed boundary could be useful, e.g., in the study of optimization of forms (see the books $[32,33]$ and the references therein). The statement below, which follows from the preceding results, shows, in particular, that the energy functional (71) for problem (2) can be approximately, but with advanced accuracy, calculated with the help of the model Dirichlet problem (74) or equation (82) (see also Remark 7); the corresponding energy functionals are simpler and look like this:

$$
\begin{aligned}
\mathcal{E}_{\varepsilon}^{\#}\left(u_{\varepsilon}^{\#} ; \Omega_{\varepsilon}^{\#}\right) & =\frac{1}{2} \int_{\Omega_{\varepsilon}^{\#}}\left|\nabla_{x} u_{\varepsilon}^{\#}(x)\right|^{2} d x-\int_{\Omega_{\varepsilon}^{\#}} f(x) u_{\varepsilon}^{\#}(x) d x, \\
\mathcal{E}_{\mathcal{A}}\left(v ; \vartheta_{\alpha}(\Xi)\right) & =\frac{1}{2}\left(\mathcal{A}\left(\vartheta_{\alpha}(\Xi)\right) v, v\right)_{\Omega_{\varepsilon}^{\#}}-(f, v)_{\Omega_{\varepsilon}^{\#}} .
\end{aligned}
$$

The same results apply also to other commonly used shape functionals (see $[34,35]$ ). 
Proposition 5. Suppose that the assumptions of Propositions 1, 2, 3, or 4 are fulfilled, depending on $\alpha$. Then the modulus of the difference between the functional computed at the solution of the corresponding model problem (see the propositions indicated above) does not exceed the quantity $C_{\natural} \varkappa_{\natural}\left\|f ; V_{\sigma}^{1}(\Omega)\right\|^{2}$; here the weight index $\sigma$ is subject to condition (21) if $\alpha<\pi$ and $\sigma=1$ if $\alpha>\pi$, and

$$
\varepsilon^{\varkappa_{\natural}}= \begin{cases}\min \{2-\sigma, 3 / 2\} & \text { if } \alpha<\pi, \\ 3 / 2 & \text { if } \alpha \in(\pi, 2 \pi), \\ -\delta+3 / 2 & \text { if } \alpha=2 \pi,\end{cases}
$$

where $\delta>0$ is an arbitrary positive number. The constant $C_{\natural}$ depends on $\sigma$ and $\delta$, but is independent of the parameter $\varepsilon \in\left(0, \varepsilon_{0}\right]$ and of the function $f$.

The approach developed in [3] makes it possible to compare the spectrum of the initial problem with that of the model problems.

\section{REFERENCES}

[1] E. Sánchez-Palencia, Nonhomogeneous media and vibration theory, Lecture Notes in Phys., vol. 127, Springer-Verlag, Berlin-New York, 1980. MR0578345 (82j:35010)

[2] E. Sánchez-Palencia and P. Suquet, Friction and homogenization of a boundary, Free Boundary Problems: Theory and Applications (Montecatini, 1981) (A. Fasano, M. Primicerio, eds.), Vols. I, II, Res. Notes in Math., vol. 78, Pitman, Boston, MA-London, 1983, pp. 561-571. MR0714936 (86b:73036)

[3] S. A. Nazarov, Binomial asymptotic behavior of solutions of spectral problems with singular perturbations, Mat. Sb. 181 (1990), no. 3, 291-320; English transl., Math. USSR-Sb. 69 (1991), no. 2, 307-340. MR1049991 (91d:35160)

[4] Y. Achdou and O. Pironneau, Domain decomposition and wall-laws, C. R. Acad. Sci. Paris Sér. I Math. 320 (1995), no. 5, 541-547. MR1322334 (95k:76087)

[5] B. Mohammadi, O. Pironneau, and F. Valentin, Rough boundaries and wall laws, Internat. J. Numer. Methods Fluids 27 (1998), no. 1-4, Special Issue, 169-177. MR1602088

[6] W. Jäger and A. Micelič, On the roughness-induced effective boundary conditions for an incompressible viscous flow, J. Differential Equations 170 (2001), no. 1, 96-122. MR1813101 (2002b:76049)

[7] M. Lobo and M. Pérez, Local problems for vibrating systems with concentrated masses: a review, C. R. Mecanique 331 (2003), 303-317.

[8] Y. Amirat, G. A. Chechkin, and R. R. Gadyl'shin, Asymptotics of simple eigenvalues and eigenfunctions for the Laplace operator in a domain with oscillating boundary, Zh. Vychisl. Mat. Mat. Fiz. 46 (2006), no. 1, 102-115; English transl., Comput. Math. Math. Phys. 46 (2006), no. 1, 97-110. MR2239730 (2007d:35009)

[9] I. S. Zorin and S. A. Nazarov, The boundary effect in the bending of a thin three-dimensional plate, Prikl. Mat. Mekh. 53 (1989), no. 4, 642-650; English transl., J. Appl. Math. Mech. 53 (1989), no. 4, 500-507 (1990). MR1022416 (90m:73045)

[10] - Two-term asymptotics of the solution to the problem on longitudinal deformation of a plate fixed along the boundary, Vychisl. Mekh. Deform. Tverd. Tela Vyp. 2 (1992), 10-21. (Russian)

[11] S. A. Nazarov, Asymptotic behavior of the solution of the Dirichlet problem in an angular domain with a periodically changing boundary, Mat. Zametki 49 (1991), no. 5, 86-96; English transl., Math. Notes 49 (1991), no. 5-6, 502-509. MR1137177 (92g:35062)

[12] M. I. Vishik and L. A. Lyusternik, Regular degeneration and boundary layer for linear differential equations with small parameter, Uspekhi Mat. Nauk (N.S.) 12 (1957), no. 5, 3-122. (Russian) MR0096041 (20:2539)

[13] V. G. Maz'ya, S. A. Nazarov, and B. A. Plamenevskiŭ, Asymptotic theory of elliptic boundary value problems in singularly perturbed domains, Tbilis. Gos. Univ., Tbilisi, 1981; English transl., Birkhäuser Verlag, Basel, 2000; German transl., Akademie-Verlag, Berlin, 1991. MR1779977 (2001e:35044a); MR1779978 (2001e:35044b); MR1186211 (93j:35025)

[14] S. A. Nazarov, The Vishik-Lyusternik method for elliptic boundary value problems in regions with conic points. I. Problem in a cone, Sibirsk. Mat. Zh. 22 (1981), no. 4, 142-163; English transl., Siberian Math. J. 22 (1981), no. 4, 594-611 (1982). MR0624412 (83m:35046a)

[15] _. The Vishik-Lyusternik method for elliptic boundary value problems in regions with conic points. II. Problem in a bounded domain, Sibirsk. Mat. Zh. 22 (1981), no. 5, 132-152; English transl., Siberian Math. J. 22 (1981), no. 5, 753-769 (1982). MR0632823 (83m:35046b) 
[16] M. Van Dyke, Perturbation methods in fluid mechanics, Parabolic Press, Stanford, CA, 1975. MR0416240 (54:4315)

[17] A. M. Il'in, Matching of asymptotic expansions of solutions of boundary value problems, "Nauka", Moscow, 1989; English transl., Transl. Math. Monogr., vol. 102, Amer. Math. Soc., Providence, RI, 1992. MR1007834 (90i:35062); MR1182791 (93g:35016)

[18] O. A. Ladyzhenskaya, Boundary value problems of mathematical physics, "Nauka", Moscow, 1973; English transl., Appl. Math. Sci., vol. 49, Springer-Verlag, New York, 1985. MR0599579 (58:29032); MR0793735 (87f:35001)

[19] F. A. Berezin and L. D. Faddeev, Remark on the Schrödinger equation with singular potential, Dokl. Akad. Nauk SSSR 137 (1961), no. 5, 1011-1014; English transl. in Soviet Math. Dokl. 2 (1961). MR0129309 (23:B2345)

[20] B. S. Pavlov, The theory of extensions, and explicitly solvable models, Uspekhi Mat. Nauk 42 (1987), no. 6, 99-131; English transl. in Russian Math. Surveys 42 (1987). MR0933997 (89b:47009)

[21] S. A. Nazarov, Asymptotic conditions at a point, selfadjoint extensions of operators and the method of matched asymptotic expansions, Trudy S.-Peterburg. Mat. Obshch. 5 (1998), 112-183; English transl., Proceedings of the St. Petersburg Mathematical Society, Vol. 5, Amer. Math. Soc. Transl. Ser. 2, vol. 193, Amer. Math. Soc., Providence, RI, 1999, pp. 77-125. MR1736907 (2000m:35016)

[22] - On the three-dimensionality effect near the tip of a crack in a thin plate, Prikl. Mat. Mekh. 55 (1991), no. 3, 500-510; English transl., J. Appl. Math. Mech. 55 (1991), no. 3, 407-415 (1992). MR1134619 (92k:73061)

[23] _ Three-dimensional effects at plate crack tips, C. R. Acad. Sci. Paris Sér. 2, 314 (1992), 995-1000.

[24] V. A. Kondrat'ev, Boundary value problems for elliptic equations in domains with conical or angular points, Trudy Moskov. Mat. Obshch. 16 (1967), 209-292; English transl. in Trans. Moscow Math. Soc. 1967 (1968). MR0226187 (37:1777)

[25] V. G. Maz'ya and B. A. Plamenevskiॅ,, The coefficients in the asymptotics of solutions of elliptic boundary value problems with conical points, Math. Nachr. 76 (1977), 29-60. (Russian) MR0601608 (58:29176)

[26] S. A. Nazarov, Polynomial property of selfadjoint elliptic boundary value problems, and the algebraic description of their attributes, Uspekhi Mat. Nauk 54 (1999), no. 5, 77-142; English transl., Russian Math. Surveys 54 (1999), no. 5, 947-1014. MR1741662 (2001k:35073)

[27] S. A. Nazarov and B. Plamenevskiu, Elliptic problems in domains with piecewise smooth boundaries, "Nauka", Moscow, 1991; English transl., Walter de Gruyter, Berlin-New York, 1994. MR1283387 (95h:35001)

[28] S. A. Nazarov and Yu. A. Romashev, Change of the intensity coefficient under failure of a bridge between collinear cracks, Izv. Akad. Nauk Arm. SSR Mekh. 35 (1982), no. 4, 30-40. (Russian)

[29] T. Kato, Perturbation theory for linear operators, 2nd ed., Grundlehren Math. Wiss., Bd. 132, Springer-Verlag, Berlin-New York, 1976. MR0407617 (53:11389)

[30] S. A. Nazarov, Selfadjoint extensions of the operator of the Dirichlet problem in weighted function spaces, Mat. Sb. (N.S.) 137 (179) (1988), no. 2, 224-241; English transl., Math. USSR-Sb. 65 (1990), no. 1, 229-247. MR0971695 (89k:35099)

[31] M. Sh. Birman and G. E. Skvortsov, On square summability of highest derivatives of the solution of the Dirichlet problem in a domain with piecewise smooth boundary, Izv. Vyssh. Uchebn. Zaved. Mat. 1962, no. 5, 12-21. (Russian) MR0145196 (26:2731)

[32] J. Sokolowski and J.-P. Zolésio, Introduction to shape optimization. Shape sensitivity analysis, Springer Ser. Comput. Math., vol. 16, Springer-Verlag, Berlin, 1992. MR1215733 (94d:49002)

[33] M. C. Delfour and J.-P. Zolésio, Shapes and geometries. Analysis, differential calculus, and optimization, Adv. in Design and Control, vol. 4, SIAM, Philadelphia, PA, 2001. MR1855817 (2002i:49002)

[34] S. A. Nazarov and J. Sokolowski, Asymptotic analysis of shape functionals, J. Math. Pures Appl. (9) 82 (2003), no. 2, 125-196. MR1976204 (2004c:35015)

[35] _ Self-adjoint extensions for the Neumann Laplacian and applications, Acta Math. Sin. (Engl. Ser.) 22 (2006), no. 3, 879-906. MR2220182 (2007a:49073)

Institute of Mechanical Engineering Problems, Bol'shoi Prospekt V.O. 61, St. Petersburg 199178, Russia

E-mail address: serna@snark.ipme.ru

Received 10/OCT/2006 